\title{
Global exponential stability of positive almost periodic solutions for a class of two-layer Gilpin-Ayala predator-prey model with time delays
}

Kaihong Zhao*

"Correspondence:
zhaokaihongs@126.com
Department of Applied
Mathematics, Kunming University of
Science and Technology, Kunming,
P.R. China

"Correspondence:

zhaokaihongs@126.com

Mathematics, Kunming University of P.R. China

\begin{abstract}
This paper mainly considers a class of two-layer Gilpin-Ayala predator-prey models with time delays. By means of Mawhin's continuation theorem of coincidence degree theory, some new sufficient criteria for the existence of positive almost periodic solutions have been established. We also obtain the global exponential stability of the positive almost periodic solution for this system by constructing appropriate Lyapunov functionals and smart transformations. As an application, an example is given to illustrate the validity of our main results.
\end{abstract}

MSC: 34K14; 34D23; 37N25

Keywords: Two-layer Gilpin-Ayala predator-prey model; Positive almost periodic solutions; Global exponential stability; Coincidence degree theory; Lyapunov functional

\section{Introduction}

In 1973, Ayala and Gilpin et al. [1] proposed the following model for studying the dynamics of competition in the fruit fly:

$$
\left\{\begin{array}{l}
x^{\prime}(t)=r_{1} x(t)\left[1-\left(\frac{x(t)}{K_{1}}\right)^{\theta_{1}}-a_{12} \frac{y(t)}{K_{2}}\right] \\
y^{\prime}(t)=r_{2} y(t)\left[1-a_{21} \frac{x(t)}{K_{1}}-\left(\frac{y(t)}{K_{2}}\right)^{\theta_{2}}\right]
\end{array}\right.
$$

where $r_{i}$ is the intrinsic rate of growth of species, $K_{i}$ is the environment carrying capacity of species $i$ in the absence of competition, $\theta_{i}$ provides a nonlinear measure of interspecific interference, and $a_{i j}$ provides a measure of interspecific interference. In the same year, Gilpin and Ayala presented a more realistic and complicated competition model in the literature [2] as follows:

$$
x_{i}^{\prime}(t)=r_{i} x_{i}(t)\left[1-\left(\frac{x_{i}(t)}{K_{i}}\right)^{\theta_{i}}-\sum_{j=1, j \neq i}^{n} a_{i j}(t) \frac{x_{j}(t)}{K_{j}}\right], \quad i=1,2, \ldots, n,
$$

\section{Springer}


where $x_{i}(t)$ is the $i$ th-species population density of at time $t, r_{i}$ is the $i$ th-species intrinsic exponential growth rate, $K_{i}$ is the $i$ th-species environment carrying capacity in the absence of competition, $\theta_{i}$ provides a nonlinear measure of intraspecific interference, and $a_{i j}(t)$ $(i \neq j)$ is the interspecific competition rate between the $i$ th species and the $j$ th species at time $t$.

It is generally called to the Gilpin-Ayala population dynamics model such as (1.1) and (1.2). Compared with the Lotka-Volterra population model, the Gilpin-Ayala population model is important and essential due to its wide applications and advantages. In fact, it is easy to see that the rate of change in the size of each species is a nonlinear function of the sizes of the interacting species in the Gilpin-Ayala population model. However, the rate of change in the size of each species is a linear function of the sizes of the interacting species in the Lotka-Volterra population model. In addition, when the value of all nonlinear measure of interspecific interference is equal to 1, the Gilpin-Ayala population model is changed into the Lotka-Volterra population model. Therefore, as soon as it was put forward, the Gilpin-Ayala models have been widely focused and deeply studied by many scholars. For example, in [3], the author proved that system (1.1) is globally stable while $\theta_{i} \geq 1$ and $\theta_{i}<1(i=1,2)$. In [4], the authors discussed the structure and global stability of equilibria for system (1.1) with infinite delay. In addition, $\mathrm{Li}$ and $\mathrm{Lu}$ [5] studied the following Gilpin-Ayala population model, more complicated than (1.2):

$$
\left\{\begin{aligned}
x_{i}^{\prime}(t) & =x_{i}(t)\left[r_{i}(t)-\sum_{j=1}^{n} a_{i j}(t) x_{j}^{\alpha_{i j}}(t)\right], \quad i=1,2, \ldots, m, \\
x_{i}^{\prime}(t) & =x_{i}(t)\left[-r_{i}(t)+\sum_{j=1}^{m} a_{i j}(t) x_{j}^{\alpha_{i j}}(t)-\sum_{j=m+1}^{n} a_{i j}(t) x_{j}^{\alpha_{i j}}(t)\right], \\
i & =m+1, \ldots, n .
\end{aligned}\right.
$$

They obtained sufficient conditions for the existence of a unique globally attractive periodic solution of system (1.3). For more work on the Gilpin-Ayala population model, one could refer to [6-16] and the references cited therein.

Motivated by the above discussion, in this paper, we mainly study the two-layer GilpinAyala predator-prey model with time delays described by

$$
\left\{\begin{aligned}
x_{i}^{\prime}(t)= & x_{i}(t)\left[r_{i}(t)-\sum_{k=1}^{n} a_{i k}(t) x_{k}^{\alpha_{i k}}(t)-\sum_{l=1}^{m} b_{i l}(t) x_{n+l}^{\beta_{i l}}(t)\right. \\
& \left.-\sum_{k=1}^{n} c_{i k}(t) x_{k}^{\gamma_{i k}}\left(t-\tau_{i k}(t)\right)-\sum_{l=1}^{m} d_{i l}(t) x_{n+l}^{\delta_{i l}}\left(t-\sigma_{i l}(t)\right)\right], \\
x_{n+j}^{\prime}(t)= & x_{n+j}(t)\left[-\hat{r}_{j}(t)+\sum_{k=1}^{n} \hat{a}_{k j}(t) x_{k}^{\hat{x}_{k j}}(t)-\sum_{l=1}^{m} \hat{b}_{l j}(t) x_{n+l}^{\hat{\beta}_{l j}}(t)\right. \\
& \left.+\sum_{k=1}^{n} \hat{c}_{k j}(t) x_{k}^{\hat{\gamma}_{k j}}\left(t-\hat{\tau}_{k j}(t)\right)-\sum_{l=1}^{m} \hat{d}_{l j}(t) x_{n+l} \hat{\delta}_{l j}\left(t-\hat{\sigma}_{l j}(t)\right)\right],
\end{aligned}\right.
$$

where $i=1,2, \ldots, n, j=1,2, \ldots, m, x_{i}(t)(i=1,2, \ldots, n)$ and $x_{n+j}(t)(j=1,2, \ldots, m)$ stand for the population density of $i$ th prey and $j$ th predator at time $t$, respectively. $r_{i}(i=1,2, \ldots, n)$ and $\hat{r}_{j}(t)(j=1,2, \ldots, m)$ present the $i$ th prey natural growth rate and the $j$ th predator natural death rate at time $t$, respectively. $a_{i k}(t), c_{i k}(t), \hat{b}_{l j}(t)$ and $\hat{d}_{l j}(t)(i, k=1,2, \ldots, n$; $j, l=1,2, \ldots, m)$ stand for the intraspecific competition rate between preys at time $t . b_{i l}(t)$ and $d_{i l}(t)(i=1,2, \ldots, n ; l=1,2, \ldots, m)$ are the predation rate of $l$ th predator on $i$ th prey at time $t . \hat{a}_{k j}(t)$ and $\hat{c}_{k j}(k=1,2, \ldots, n ; j=1,2, \ldots, m)$ are the conversion rate of $k$ th prey to $j$ th predator at time $t . \tau_{i k}(t), \sigma_{i l}(t), \hat{\tau}_{k j}(t)$ and $\sigma_{l j}(t)(i, k=1,2, \ldots, n ; j, l=1,2, \ldots, m)$ are the discrete time delay at time $t$. The constants $\alpha_{i k}, \beta_{i l}, \gamma_{i k}, \delta_{i l}, \hat{\alpha}_{k j}, \hat{\beta}_{l j}, \hat{\gamma}_{k j}$ and $\hat{\delta}_{l j}(i, k=1,2, \ldots, n$; $j, l=1,2, \ldots, m)$ provide a nonlinear measure of intraspecific interference. 
To the best of our knowledge, few authors have considered the existence and global exponential stability of positive almost periodic solutions for model (1.4). However, the previous papers deal with the permanence, periodic solution and their global attractivity of the Gilpin-Ayala population models. Indeed, the effects of an almost periodically varying environment are important for evolutionary theory as the selective forces on systems in a fluctuating environment differ from those in a stable environment. Therefore, we will establish some sufficient conditions of existence and global exponential stability of positive almost periodic solutions for model (1.4).

The remain of this paper is organized as follows. In Sect. 2, we give some notations and lemmas. In Sect. 3, some new sufficient criteria for the existence of positive almost periodic solutions have been established by means of Mawhin's continuation theorem of coincidence degree theory. In Sect. 4, we also obtain the global exponential stability of the positive almost periodic solution of this system by constructing appropriate Lyapunov functionals and inequality techniques. As applications, an example and its simulation are given to illustrate the validity of our main results in Sect. 5. Finally, we conclude with the main results and their biological meaning in Sect. 6.

\section{Preliminaries}

In this section, we first recall some basic definitions and lemmas which are used in what follows.

Definition 2.1 ([17]) Let $u(t): \mathbb{R} \rightarrow \mathbb{R}$ be continuous in $t . u(t)$ is said to be almost periodic on $\mathbb{R}$, if, for any $\epsilon>0$, the set $K(u, \epsilon)=\{\delta:|u(t+\delta)-u(t)|<\epsilon, \forall t \in \mathbb{R}\}$ is relatively dense, that is, for any $\epsilon>0$, it is possible to find a real number $l(\epsilon)>0$, for any interval with length $l(\epsilon)$, there exists a number $\delta=\delta(\epsilon)$ in this interval such that $|u(t+\delta)-u(t)|<\epsilon, \forall t \in \mathbb{R}$.

Definition 2.2 A solution $\left(x_{1}(t), \ldots, x_{n}(t), x_{n+1}(t), \ldots, x_{n+m}(t)\right)^{T}$ of $(1.1)$ is called an almost periodic solution if and only if $x_{i}(t)$ and $x_{n+j}(t)(i=1,2, \ldots, n ; j=1,2, \ldots, m)$ are almost periodic.

For convenience, we denote $\mathrm{AP}(\mathbb{R})$ is the set of all real valued, almost periodic functions on $\mathbb{R}$. For $f \in \mathrm{AP}(\mathbb{R})$, define

$$
\wedge(f)=\left\{\tilde{\lambda} \in \mathbb{R}: \lim _{T \rightarrow \infty} \frac{1}{T} \int_{0}^{T} f(s) e^{-i \tilde{\lambda} s} d s \neq 0\right\}
$$

and

$$
\bmod (f)=\left\{\sum_{i=1}^{N} n_{i} \tilde{\lambda}_{i}: n_{i} \in Z, N \in N^{+}, \tilde{\lambda}_{i} \in \wedge(f)\right\}
$$

to be the set of Fourier exponents and the module of $f$, respectively. $m(f)=\frac{1}{T} \int_{0}^{T} f(s) d s$ be the mean value of $f$ on interval $[0, T]$, where $T>0$ is a constant. Clearly, $m(f)$ depends on $T . m[f]=\lim _{T \rightarrow \infty} \frac{1}{T} \int_{0}^{T} f(s) d s$. Suppose that $f(t, \phi)$ is almost periodic in $t$, uniformly with respect to $\phi \in C([-\sigma, 0], \mathbb{R})$, let $K(f, \epsilon, S)$ denote the set of $\epsilon$-almost periods with respect to $S \subset C([-\sigma, 0], \mathbb{R})$ and $l(\epsilon, S)$ denote the length of inclusion interval. 
Lemma 2.1 ([17]) Suppose that $f$ and $g$ are almost periodic. Then the following statements are equivalent:

(i) $\bmod (f) \supset \bmod (g)$,

(ii) for any sequence $\left\{t_{n}^{*}\right\}$, if $\lim _{n \rightarrow \infty} f\left(t+t_{n}^{*}\right)=f(t)$ for each $t \in \mathbb{R}$, then there exists a subsequence $\left\{t_{n}\right\} \subseteq\left\{t_{n}^{*}\right\}$ such that $\lim _{n \rightarrow \infty} g\left(t+t_{n}\right)=f(t)$ for each $t \in \mathbb{R}$.

Lemma 2.2 ([17]) If $u \in \mathrm{AP}(\mathbb{R})$, then $\int_{t-\tau}^{t} u(s) d s$ is almost periodic.

Lemma 2.3 ([17]) If $u(t) \in \mathrm{AP}(\mathbb{R})$, then $u(t)$ is bounded on $\mathbb{R}$.

Lemma 2.4 ([18]) If $f(t) \in \mathrm{AP}(\mathbb{R})$, then there exists $t_{0} \in \mathbb{R}$ such that $f\left(t_{0}\right)=m(f)$.

Lemma $2.5([18])$ Assume that $u(t) \in \mathrm{AP}(\mathbb{R}) \cap C^{1}(\mathbb{R}, \mathbb{R})$, then there exist two point sequences $\left\{\xi_{k}\right\}_{k=1}^{\infty}$ and $\left\{\eta_{k}\right\}_{k=1}^{\infty}$ such that $u^{\prime}\left(\xi_{k}\right)=u^{\prime}\left(\eta_{k}\right)=0, \lim _{k \rightarrow \infty} \xi_{k}=\infty, \lim _{k \rightarrow \infty} \eta_{k}=-\infty$.

Lemma 2.6 ([18]) Assume that $u(t) \in \mathrm{AP}(\mathbb{R}) \cap C^{1}(\mathbb{R}, \mathbb{R})$, then $u(t)$ falls into one of the following four cases:

(i) There are $\xi, \eta \in \mathbb{R}$ such that $u(\xi)=\sup _{t \in \mathbb{R}} u(t)$ and $u(\eta)=\inf _{t \in \mathbb{R}} u(t)$. In this case, $u^{\prime}(\xi)=u^{\prime}(\eta)=0$.

(ii) There are no $\xi, \eta \in \mathbb{R}$ such that $u(\xi)=\sup _{t \in \mathbb{R}} u(t)$ and $u(\eta)=\inf _{t \in \mathbb{R}} u(t)$. In this case, for all $\epsilon>0$, there exist two points $\xi, \eta \in \mathbb{R}$ such that $u^{\prime}(\xi)=u^{\prime}(\eta)=0$, $u(\xi)>\sup _{t \in \mathbb{R}} u(t)-\epsilon$ and $u(\eta)<\inf _{t \in \mathbb{R}} u(t)+\epsilon$.

(iii) There is a $\xi \in \mathbb{R}$ such that $u(\xi)=\sup _{t \in \mathbb{R}} u(t)$ and there is no $\eta \in \mathbb{R}$ such that $u(\eta)=\inf _{t \in \mathbb{R}} u(t)$. In this case, $u^{\prime}(\xi)=0$ and for all $\epsilon>0$, there exists an $\eta \in \mathbb{R}$ such that $u^{\prime}(\eta)=0$ and $u(\eta)<\inf _{t \in \mathbb{R}} u(t)+\epsilon$.

(vi) There is an $\eta \in \mathbb{R}$ such that $u(\eta)=\inf _{t \in \mathbb{R}} u(t)$ and there is no $\xi \in \mathbb{R}$ such that $u(\eta)=\sup _{t \in \mathbb{R}} u(t)$. In this case, $u^{\prime}(\eta)=0$ and for all $\epsilon>0$, there exists an $\xi \in \mathbb{R}$ such that $u^{\prime}(\xi)=0$ and $u(\xi)>\sup _{t \in \mathbb{R}} u(t)-\epsilon$.

For the sake of convenience, we denote $v^{l}=\inf _{t \in \mathbb{R}} v(t), v^{M}=\sup _{t \in \mathbb{R}} v(t)$, here $v(t)$ is a continuous almost periodic function on $\mathbb{R}$. For simplicity, we need to introduce some notations as follows:

$$
\begin{aligned}
& l_{i}^{+}=\left(\frac{r_{i}^{M}}{a_{i i}^{l}}\right)^{\frac{1}{\alpha_{i i}}}, \quad l_{n+j}^{+}=\left(\frac{\sum_{k=1}^{n} \hat{a}_{k j}^{M}\left(l_{k}^{+}\right)^{\hat{\alpha}_{k j}}+\sum_{k=1}^{n} \hat{c}_{k j}^{M}\left(l_{k}^{+}\right)^{\hat{\gamma}_{k j}}}{\hat{b}_{i j}^{l}}\right)^{\frac{1}{\hat{\beta}_{j j}}}, \\
& l_{i}^{-}=\left(\frac{r_{i}^{l}-\sum_{k=1, k \neq i}^{n} a_{i k}^{M}\left(l_{k}^{+}\right)^{\alpha_{i k}}-\sum_{l=1}^{m} b_{i l}^{M}\left(l_{n+l}^{+}\right)^{\beta_{i l}}-\sum_{k=1}^{n} c_{i k}^{M}\left(l_{k}^{+}\right)^{\gamma_{i k}}-\sum_{l=1}^{m} d_{i l}^{M}\left(l_{n+l}^{+}\right)^{\delta_{i l}}}{a_{i i}^{M}}\right)^{\frac{1}{\alpha_{i i}}}, \\
& l_{n+j}^{-}=\left(\frac{\sum_{k=1}^{n} \hat{a}_{k j}^{l}\left(l_{k}^{-}\right)^{\hat{\alpha}_{k j}}+\sum_{k=1}^{n} \hat{c}_{k j}^{l}\left(l_{k}^{-}\right)^{\hat{\gamma}_{k j}}-\hat{r}_{j}^{M}-\sum_{l=1, l \neq j}^{m} \hat{b}_{l j}^{M}\left(l_{n+l}^{+}\right)^{\hat{\beta}_{l j}}-\sum_{l=1}^{m} \hat{d}_{l j}^{M}\left(l_{n+l}^{+}\right)^{\hat{\delta}_{l j}}}{\hat{b}_{j j}^{M}}\right)^{\frac{1}{\hat{\beta}_{j j}}},
\end{aligned}
$$

where $i=1,2, \ldots, n, j=1,2, \ldots, m$.

Throughout this paper, we need the following assumptions.

$\left(H_{1}\right) r_{i}(t), \hat{r}_{j}(t), a_{i k}(t), b_{i l}(t), c_{i k}(t), d_{i l}(t), \hat{a}_{k j}(t), \hat{b}_{l j}(t), \hat{c}_{k j}(t), \hat{d}_{l j}(t)(i, k=1,2, \ldots, n ; j, l=$ $1,2, \ldots, m) \in C\left(\mathbb{R}, \mathbb{R}^{+}\right)$and $\tau_{i k}(t), \sigma_{i l}(t), \hat{\tau}_{k j}(t), \sigma_{l j}(t)(i, k=1,2, \ldots, n ; j, l=1,2, \ldots$, $m) \in C\left(\mathbb{R}, \mathbb{R}_{0}^{+}\right)$are all continuous positive almost periodic functions with respect to the time variable $t$, where $\mathbb{R}^{-}=(-\infty, 0), \mathbb{R}^{+}=(0, \infty), \mathbb{R}_{0}^{+}=[0, \infty), \mathbb{R}=(-\infty,+\infty)$. 
$\left(H_{2}\right)$ The nonlinear measures of intraspecific interference $\alpha_{i k}, \beta_{i l}, \gamma_{i k}, \delta_{i l}, \hat{\alpha}_{k j}, \hat{\beta}_{l j}, \hat{\gamma}_{k j}$ and $\hat{\delta}_{l j}(i, k=1,2, \ldots, n ; j, l=1,2, \ldots, m)$ are all the positive constants, that is, $\alpha_{i k}>0$, $\beta_{i l}>0, \gamma_{i k}>0, \delta_{i l}>0, \hat{\alpha}_{k j}>0, \hat{\beta}_{l j}>0, \hat{\gamma}_{k j}>0, \hat{\delta}_{l j}>0$.

$\left(H_{3}\right) r_{i}^{l}>\sum_{k=1, k \neq i}^{n} a_{i k}^{M}\left(l_{k}^{+}\right)^{\alpha_{i k}}+\sum_{l=1}^{m} b_{i l}^{M}\left(l_{n+l}^{+}\right)^{\beta_{i l}}+\sum_{k=1}^{n} c_{i k}^{M}\left(l_{k}^{+}\right)^{\gamma_{i k}}+\sum_{l=1}^{m} d_{i l}^{M}\left(l_{n+l}^{+}\right)^{\delta_{i l}}$ and $\sum_{k=1}^{n} \hat{a}_{k j}^{l}\left(l_{k}^{-}\right)^{\hat{\alpha}_{k j}}+\sum_{k=1}^{n} \hat{c}_{k j}^{l}\left(l_{k}^{-}\right)^{\hat{\gamma}_{k j}}>\hat{r}_{j}^{M}+\sum_{l=1, l \neq j}^{m} \hat{b}_{l j}^{M}\left(l_{n+l}^{+}\right)^{\hat{\beta}_{l j}}+\sum_{l=1}^{m} \hat{d}_{l j}^{M}\left(l_{n+l}^{+}\right)^{\hat{\delta}_{l j}, i=}$ $1,2, \ldots, n ; j=1,2, \ldots, m$.

\section{Existence of positive almost periodic solution}

In this section, by using Mawhin's continuation theorem, we shall show the existence of positive almost periodic solutions of (1.1).

Let $X$ and $Z$ be Banach spaces. $L: \operatorname{Dom}(L) \subset X \rightarrow Z$ be a linear mapping and $N: X \times$ $[0,1] \rightarrow Z$ is a continuous mapping. The mapping $L$ will be called a Fredholm mapping of index zero if $\operatorname{dim} \operatorname{Ker}(L)=\operatorname{codim} \operatorname{Im}(L)<\infty$ and $\operatorname{Im}(L)$ is closed in $Z$. If $L$ is a Fredholm mapping of index zero, then there exist continuous projectors $P: X \rightarrow X$ and $Q: Z \rightarrow Z$ such that $\operatorname{Im}(P)=\operatorname{Ker}(L)$ and $\operatorname{Ker}(Q)=\operatorname{Im}(L)=\operatorname{Im}(I-Q)$, and $X=\operatorname{Ker}(L) \oplus \operatorname{Ker}(P), Z=$ $\operatorname{Im}(L) \oplus \operatorname{Im}(Q)$. It follows that $\left.L\right|_{\operatorname{Dom}(L) \cap \operatorname{Ker}(P)}:(I-P) X \rightarrow \operatorname{Im}(L)$ is invertible and its inverse is denoted by $K_{P}$. If $\Omega$ is a bounded open subset of $X$, the mapping $N$ is called $L$-compact on $\bar{\Omega} \times[0,1]$, if $Q N(\bar{\Omega} \times[0,1])$ is bounded and $K_{p}(I-Q) N: \bar{\Omega} \times[0,1] \rightarrow X$ is compact, where $I$ is the identity. Because $\operatorname{Im}(Q)$ is isomorphic to $\operatorname{Ker}(L)$, there exists an isomorphism $J: \operatorname{Im}(Q) \rightarrow \operatorname{Ker}(L)$.

Let $L$ be a Fredholm linear mapping with index zero and let $N$ be a $L$-compact mapping on $\bar{\Omega}$. Define mapping $F: \operatorname{Dom}(L) \cap \bar{\Omega} \rightarrow Z$ by $F=L-N$. If $L x \neq N x$ for all $x \in \operatorname{Dom}(L) \cap \partial \Omega$, then by using $P, Q, K_{P}, J$ defined above, the coincidence degree of $F$ in $\Omega$ with respect to $L$ is defined by

$$
\operatorname{deg}_{L}(F, \Omega)=\operatorname{deg}\left(I-P-\left(J^{-1} Q+K_{P}(I-Q)\right) N, \Omega, 0\right)
$$

where $\operatorname{deg}(g, \Gamma, p)$ is the Leray-Schauder degree of $g$ at $p$ relative to $\Gamma$.

The Mawhin's continuous theorem [19], p. 40, is given as follows:

Lemma 3.1 ([19]) Let $L$ be a Fredholm mapping of index zero and $N$ be L-compact on $\bar{\Omega} \times[0,1]$. Assume

(a) for each $\lambda \in(0,1)$, every solution $x$ of $L x=\lambda N(x, \lambda)$ is such that $x \notin \partial \Omega \cap \operatorname{Dom}(L)$;

(b) $Q N(x, 0) \neq 0$ for each $x \in \partial \Omega \cap \operatorname{Ker}(L)$;

(c) $\operatorname{deg}(J Q N(x, 0), \Omega \cap \operatorname{Ker}(L), 0) \neq 0$.

Then $L x=N(x, 1)$ has at least one solution in $\bar{\Omega} \cap \operatorname{Dom}(L)$.

By making the substitution $x_{i}(t)=e^{u_{i}(t)}(i=1,2, \ldots, n), x_{n+j}(t)=e^{u_{n+j}(t)}(j=1,2, \ldots, m)$, then system (1.4) is rewritten in the form of

$$
\left\{\begin{aligned}
u_{i}^{\prime}(t)= & r_{i}(t)-\sum_{k=1}^{n} a_{i k}(t) e^{\alpha_{i k} u_{k}(t)}-\sum_{l=1}^{m} b_{i l}(t) e^{\beta_{i l} u_{n+l}(t)} \\
& -\sum_{k=1}^{n} c_{i k}(t) e^{\gamma_{i k} u_{k}\left(t-\tau_{i k}(t)\right)} \\
& -\sum_{l=1}^{m} d_{i l}(t) e^{\delta_{i l} u_{n+l}\left(t-\sigma_{i l}(t)\right)}, \quad i=1,2, \ldots, n, \\
u_{n+j}^{\prime}(t)= & -\hat{r}_{j}(t)+\sum_{k=1}^{n} \hat{a}_{k j}(t) e^{\hat{\alpha}_{k j} u_{k}(t)}-\sum_{l=1}^{m} \hat{b}_{l j}(t) e^{\hat{\beta}_{l j} u_{n+l}(t)} \\
& +\sum_{k=1}^{n} \hat{c}_{k j}(t) e^{\hat{\gamma}_{k j} u_{k}\left(t-\hat{\tau}_{k j}(t)\right)} \\
& -\sum_{l=1}^{m} \hat{d}_{l j}(t) e^{\hat{\delta}_{l j} u_{n+l}\left(t-\hat{\sigma}_{l j}(t)\right)}, \quad j=1,2, \ldots, m .
\end{aligned}\right.
$$


Let $X=Z=V_{1} \oplus V_{2}$, where

$$
\begin{aligned}
& V_{1}=\left\{w(t)=\left(w_{1}(t), w_{2}(t), \ldots, w_{n+m}(t)\right)^{T}: w_{k}(t) \in \mathrm{AP}(\mathbb{R}), \bmod \left(w_{k}(t)\right) \subset \bmod \left(F_{k}(t)\right),\right. \\
& \left.\forall \tilde{\lambda}_{k} \in \wedge\left(w_{k}(t)\right) \text { satisfies }\left|\tilde{\lambda}_{k}\right|>\beta, k=1,2, \ldots, n+m\right\}, \\
& V_{2}=\left\{w(t) \equiv\left(c_{1}, c_{2}, \ldots, c_{n+m}\right) \in \mathbb{R}^{n+m}\right\}, \\
& F_{i}(t)=r_{i}(t)-\sum_{k=1}^{n} a_{i k}(t) e^{\alpha_{i k} \phi_{k}(0)}-\sum_{l=1}^{m} b_{i l}(t) e^{\beta_{i l} \phi_{n+l}(0)}-\sum_{k=1}^{n} c_{i k}(t) e^{\gamma_{i k} \phi_{k}\left(-\tau_{i k}(t)\right)} \\
& \quad-\sum_{l=1}^{m} d_{i l}(t) e^{\delta_{i l} \phi_{n+l}\left(-\sigma_{i l}(t)\right)}, \quad i=1,2, \ldots, n, \\
& F_{n+j}(t)=-\hat{r}_{j}(t)+\sum_{k=1}^{n} \hat{a}_{k j}(t) e^{\hat{\alpha}_{k j} \phi_{k}(0)}-\sum_{l=1}^{m} \hat{b}_{l j}(t) e^{\hat{\beta}_{l j} \phi_{n+l}(0)}+\sum_{k=1}^{n} \hat{c}_{k j}(t) e^{\hat{\gamma}_{k j} \phi_{k}\left(-\hat{t}_{k j}(t)\right)} \\
& \quad-\sum_{l=1}^{m} \hat{d}_{l j}(t) e^{\hat{\delta}_{l j} \phi_{n+l}\left(-\hat{\sigma}_{l j}(t)\right)}, \quad j=1,2, \ldots, m,
\end{aligned}
$$

in which $\phi_{k} \in C([-\sigma, 0], \mathbb{R}), k=1,2, \ldots, n+m, \sigma=\max _{1 \leq i, k \leq n, 1 \leq j, l \leq m} \sup _{t \in \mathbb{R}}\left\{\tau_{i k}(t), \sigma_{i l}(t)\right.$, $\left.\hat{\tau}_{i k}(t), \hat{\sigma}_{i l}(t)\right\}$, and $\beta$ is a given constant. Define the norm

$$
\|w\|=\max _{1 \leq k \leq n+m} \sup _{t \in \mathbb{R}}\left|w_{k}(t)\right|, \quad \forall w=\left(w_{1}, w_{2}, \ldots, w_{n+m}\right)^{T} \in X=Z
$$

Lemma 3.2 $X=Z$ is a Banach space equipped with the norm $\|\cdot\|$ defined by (3.2).

Proof Assume that $\left\{w^{\{k\}}=\left(w_{1}^{\{k\}}, w_{2}^{\{k\}}, \ldots, w_{n+m}^{\{k\}}\right)^{T}\right\} \subset V_{1}$ converge to $\bar{w}=\left(\bar{w}_{1}, \bar{w}_{2}, \ldots, \bar{w}_{n+m}\right)^{T}$, that is, $w_{h}^{\{k\}} \rightarrow \bar{w}_{h}$, as $k \rightarrow \infty, h=1,2, \ldots, n+m$. Then it is easy to show that $\bar{w}_{h} \in \operatorname{AP}(\mathbb{R})$ and $\bmod \left(\bar{w}_{h}\right) \in \bmod \left(F_{h}\right)$. For any $\left|\widetilde{\lambda}_{h}\right| \leq \beta$, we have

$$
\lim _{T \rightarrow \infty} \frac{1}{T} \int_{0}^{T} w_{h}^{\{k\}}(t) e^{-\tilde{\lambda}_{h} t} d t=0, \quad h=1,2, \ldots, n+m,
$$

therefore,

$$
\lim _{T \rightarrow \infty} \frac{1}{T} \int_{0}^{T} \bar{w}_{h}^{\{k\}}(t) e^{-i \tilde{\lambda}_{h} t} d t=0, \quad h=1,2, \ldots, n+m,
$$

which implies $\bar{w} \in V_{1}$. Then it is not difficult to see that $V_{1}$ is a Banach space equipped with the norm $\|\cdot\|$. Thus, we can easily verify that $X$ and $Z$ are Banach spaces equipped with the norm $\|\cdot\|$. The proof is complete.

Lemma 3.3 Let $L: X \rightarrow Z, L w=\frac{d w(t)}{d t}$, then $L$ is a Fredholm mapping of index zero.

Proof Clearly, $L$ is a linear operator and $\operatorname{Ker}(L)=V_{2}$. We claim that $\operatorname{Im}(L)=V_{1}$. In fact, we suppose that $z(t)=\left(z_{1}(t), z_{2}(t), \ldots, z_{n+m}(t)\right)^{T} \in \operatorname{Im}(L) \subset Z$, then there exist $z^{\{1\}}(t)=$ $\left(z_{1}^{\{1\}}(t), z_{2}^{\{1\}}(t), \ldots, z_{n+m}^{\{1\}}(t)\right)^{T} \in V_{1}$ and constant vector $z^{\{2\}}=\left\{z_{1}^{\{2\}}(t), z_{2}^{\{2\}}(t), \ldots, z_{n+m}^{\{2\}}(t)\right\} \in V_{2}$ such that $z(t)=z^{\{1\}}(t)+z^{\{2\}}$, that is, $z_{h}(t)=z_{h}^{\{1\}}(t)+z_{h}^{\{2\}}, h=1,2, \ldots, n+m$. From Lemma 2.2 
and the definitions of $z_{i}(t)$ and $z_{i}^{\{1\}}(t)$, we know that $\int_{t-\sigma}^{t} z_{h}(s) d s$ and $\int_{t-\sigma}^{t} z_{h}^{\{1\}}(s) d s$ are almost periodic functions. So we have $z_{h}^{\{2\}} \equiv 0, h=1,2, \ldots, n+m$, then $z^{\{2\}} \equiv(0,0, \ldots, 0)^{T}$, which implies $z(t) \in V_{1}$, that is, $\operatorname{Im}(L) \subset V_{1}$.

On the other hand, if $w(t)=\left(w_{1}(t), w_{2}(t), \ldots, w_{n+m}(t)\right)^{T} \in V_{1} \backslash\left\{(0,0, \ldots, 0)^{T}\right\}$, then we have $\int_{0}^{t} w_{h}(s) d s \in \mathrm{AP}(\mathbb{R}), h=1,2, \ldots, n+m$. If $\tilde{\lambda}_{h} \neq 0$, then we obtain

$$
\lim _{T \rightarrow \infty} \frac{1}{T} \int_{0}^{T}\left(\int_{0}^{t} w_{h}(s) d s\right) e^{-\tilde{\lambda}_{j} t} d t=\frac{1}{i \widetilde{\lambda}_{h}} \lim _{T \rightarrow \infty} \frac{1}{T} \int_{0}^{T} w_{h}(t) e^{-i \tilde{\lambda}_{h} t} d t
$$

$h=1,2, \ldots, n+m$. It follows that

$$
\wedge\left[\int_{0}^{t} w_{h}(s) d s-m\left(\int_{0}^{t} w_{h}(s) d s\right)\right]=\wedge\left(w_{h}(t)\right), \quad h=1,2, \ldots, n+m,
$$

hence

$$
\int_{0}^{t} w(s) d s-m\left(\int_{0}^{t} w(s) d s\right) \in V_{1} \subset X .
$$

Note that $\int_{0}^{t} w(s) d s-m\left(\int_{0}^{t} w(s) d s\right)$ is the primitive of $w(t)$ in $X$, we have $w(t) \in \operatorname{Im}(L)$, that is, $V_{1} \subset \operatorname{Im}(L)$. Therefore, $\operatorname{Im}(L)=V_{1}$.

Furthermore, one easily shows that $\operatorname{Im}(L)$ is closed in $Z$ and

$$
\operatorname{dim} \operatorname{ker}(L)=n+m=\operatorname{codim} \operatorname{Im}(L) .
$$

Therefore, $L$ is a Fredholm mapping of index zero. The proof is complete.

Lemma 3.4 Let $N: X \times(0,1) \rightarrow Z, N(u(t), \lambda)=\left(N_{1}^{u}, N_{2}^{u}, \ldots, N_{n+m}^{u}\right)^{T}$, where

$$
\begin{aligned}
N_{i}^{u}= & r_{i}(t)-a_{i i}(t) e^{\alpha_{i i} u_{i}(t)}-\lambda \sum_{k=1, k \neq i}^{n} a_{i k}(t) e^{\alpha_{i k} u_{k}(t)}-\lambda \sum_{l=1}^{m} b_{i l}(t) e^{\beta_{i l} u_{n+l}(t)} \\
& -\lambda \sum_{k=1}^{n} c_{i k}(t) e^{\gamma_{i k} u_{k}\left(t-\tau_{i k}(t)\right)}-\lambda \sum_{l=1}^{m} d_{i l}(t) e^{\delta_{i l} u_{n+l}\left(t-\sigma_{i l}(t)\right)}, \quad i=1,2, \ldots, n,
\end{aligned}
$$

and

$$
\begin{aligned}
N_{n+j}^{u}= & -\hat{r}_{j}(t)-\hat{b}_{j j}(t) e^{\hat{\beta}_{j j} u_{n+j}(t)}+\sum_{k=1}^{n} \hat{a}_{k j}(t) e^{\hat{\alpha}_{k j} u_{k}(t)}-\lambda \sum_{l=1, l \neq j}^{m} \hat{b}_{l j}(t) e^{\hat{\beta}_{l j} u_{n+l}(t)} \\
& +\sum_{k=1}^{n} \hat{c}_{k j}(t) e^{\hat{\gamma}_{k j} u_{k}\left(t-\hat{\tau}_{k j}(t)\right)}-\lambda \sum_{l=1}^{m} \hat{d}_{l j}(t) e^{\hat{\delta}_{l j} u_{n+l}\left(t-\hat{\sigma}_{l j}(t)\right)}, \quad j=1,2, \ldots, m .
\end{aligned}
$$

Define the projectors

$$
\begin{aligned}
& P: X \rightarrow Z, \quad P u=\left(m\left(u_{1}\right), m\left(u_{2}\right), \ldots, m\left(u_{n+m}\right)\right)^{T}, \\
& Q: Z \rightarrow Z, \quad Q z=\left(m\left[z_{1}\right], m\left[z_{2}\right], \ldots, m\left[z_{n+m}\right]\right)^{T} .
\end{aligned}
$$

Then $N$ is L-compact on $\bar{\Omega}$, where $\Omega$ is an open bounded subset of $X$. 
Proof Obviously, $P$ and $Q$ are continuous projectors such that

$$
\operatorname{Im}(P)=\operatorname{Ker}(L), \quad \operatorname{Im}(L)=\operatorname{Ker}(Q)
$$

It is clear that $(I-Q) V_{2}=\{(0,0, \ldots, 0)\},(I-Q) V_{1}=V_{1}$. Hence

$$
\operatorname{Im}(I-Q)=V_{1}=\operatorname{Im}(L)
$$

In view of

$$
\operatorname{Im}(P)=\operatorname{Ker}(L), \quad \operatorname{Im}(L)=\operatorname{Ker}(Q)=\operatorname{Im}(I-Q),
$$

we find that the inverse $K_{P}: \operatorname{Im}(L) \rightarrow \operatorname{Ker}(P) \cap \operatorname{Dom}(L)$ of $L_{P}$ exists and is given by

$$
K_{P}(z)=\int_{0}^{t} z(s) d s-m\left[\int_{0}^{t} z(s) d s\right]
$$

Thus,

$$
\begin{aligned}
& Q N u=\left(m\left[H_{1}^{u}\right], m\left[H_{2}^{u}\right], \ldots, m\left[H_{n+m}^{u}\right]\right)^{T}, \\
& K_{P}(I-Q) N u=\left(f\left(u_{1}\right)-Q\left(f\left(u_{1}\right)\right), f\left(u_{2}\right)-Q\left(f\left(u_{2}\right)\right), \ldots, f\left(u_{n+m}\right)-Q\left(f\left(u_{n+m}\right)\right)\right)^{T},
\end{aligned}
$$

where

$$
f\left(u_{h}\right)=\int_{0}^{t}\left(H_{h}^{u}-m\left[H_{h}^{u}\right]\right) d s, \quad h=1,2, \ldots, n+m .
$$

Clearly, $Q N$ and $(I-Q) N$ are continuous. Now we show that $K_{P}$ is also continuous. By assumptions, for any $0<\epsilon<1$, let $l_{h}\left(\epsilon_{h}, S\right)$ be the length of the inclusion interval of $K_{h}\left(F_{h}, \epsilon_{h}, S\right), h=1,2, \ldots, n+m$. Suppose that $\left\{z^{k}(t)\right\} \subset \operatorname{Im}(L)=V_{1}$ and $z^{k}(t)=$ $\left(z_{1}^{k}(t), z_{2}^{k}(t), \ldots, z_{n+m}^{k}(t)\right)^{T}$ uniformly converges to $\bar{z}(t)=\left(\bar{z}_{1}(t), \bar{z}_{2}(t), \ldots, \bar{z}_{n+m}(t)\right)^{T}$, that is $z_{h}^{k} \rightarrow \bar{z}_{h}$, as $k \rightarrow \infty, h=1,2, \ldots, n+m$. Because of $\int_{0}^{t} z^{k}(s) d s \in Z, k=1,2, \ldots$, there exists $\sigma_{h}\left(0<\sigma_{h}<\epsilon_{h}\right)$ such that $K_{h}\left(F_{h}, \sigma_{h}, S\right) \subset K_{h}\left(\int_{0}^{t} z_{h}^{k}(s) d s, \sigma_{h}\right), h=1,2, \ldots, n+m$. Let $l_{h}\left(\sigma_{h}, S\right)$ be the length of the inclusion interval of $K_{h}\left(F_{h}, \sigma_{h}, S\right), h=1,2, \ldots, n+m$ and $l_{h}=\max \left\{l_{h}\left(\epsilon_{h}, S\right), l_{h}\left(\sigma_{h}, S\right)\right\}, h=1,2, \ldots, n+m$. It is easy to see that $l_{h}$ is the length of the inclusion interval of $K_{h}\left(F_{h}, \sigma_{h}, S\right)$ and $K_{h}\left(F_{h}, \epsilon_{h}, S\right), h=1,2, \ldots, n+m$. Hence, for any $t \notin\left[0, l_{h}\right]$, there exists $\xi_{t} \in K_{h}\left(F_{h}, \sigma_{h}, S\right) \subset K_{h}\left(\int_{0}^{t} z_{h}^{k}(s) d s, \sigma_{h}, S\right)$ such that $t+\xi_{t} \in\left[0, l_{h}\right]$, $h=1,2, \ldots, n+m$. So, by the definition of almost periodic function we have

$$
\begin{aligned}
& \left\|\int_{0}^{t} z^{k}(s) d s\right\| \\
& =\max _{1 \leq h \leq n+m} \sup _{t \in \mathbb{R}}\left|\int_{0}^{t} z_{h}^{k}(s) d s\right| \\
& \quad \leq \max _{1 \leq h \leq n+m} \sup _{t \in\left[0, l_{h}\right]}\left|\int_{0}^{t} z_{h}^{k}(s) d s\right| \\
& \quad+\max _{1 \leq h \leq n+m} \sup _{t \notin\left[0, l_{h}\right]}\left|\int_{0}^{t} z_{h}^{k}(s) d s-\int_{0}^{t+\xi_{l}} z_{h}^{k}(s) d s+\int_{0}^{t+\xi_{l}} z_{h}^{k}(s) d s\right|
\end{aligned}
$$




$$
\begin{aligned}
& \leq 2 \max _{1 \leq h \leq n+m} \sup _{t \in\left[0, l_{h}\right]}\left|\int_{0}^{t} z_{h}^{k}(s) d s\right| \\
& \quad+\max _{1 \leq h \leq n+m} \sup _{t \notin\left[0, l_{h}\right]}\left|\int_{0}^{t} z_{h}^{k}(s) d s-\int_{0}^{t+\xi_{l}} z_{h}^{k}(s) d s\right| \\
& \leq 2 \max _{1 \leq h \leq n+m}\left|\int_{0}^{l_{h}} z_{h}^{k}(s) d s\right|+\max _{1 \leq h \leq n+m} \epsilon_{h} .
\end{aligned}
$$

From (3.3), we conclude that $\int_{0}^{t} z(s) d s$ is continuous, where $z(t)=\left(z_{1}(t), z_{2}(t)\right.$, $\left.\ldots, z_{n+m}(t)\right)^{T} \in \operatorname{Im}(L)$. Consequently, $K_{P}$ and $K_{P}(I-Q) N y$ are continuous. Meanwhile, we also have $\int_{0}^{t} z(s) d s$ and $K_{P}(I-P) N u$ are uniformly bounded in $\bar{\Omega}$. Further, it is not difficult to verify that $Q N(\bar{\Omega})$ is bounded and $K_{P}(I-Q) N u$ is equicontinuous in $\bar{\Omega}$. By the ArzelaAscoli theorem, we have immediately conclude that $K_{P}(I-Q) N(\bar{\Omega})$ is compact. Thus $N$ is $L$-compact on $\bar{\Omega}$. The proof is complete.

Theorem 3.1 Assume that $\left(H_{1}\right)-\left(H_{3}\right)$ hold. Then system (1.4) has at least one positive almost periodic solution.

Proof In order to use Lemma 3.1 establishing the existence of positive almost periodic solutions of (1.1), we set Banach space $X$ and $Z$ the same as those in Lemma 3.2 and set mappings $L, N, P, Q$ the same as those in Lemma 3.3 and Lemma 3.4, respectively. Then we find that $L$ is a Fredholm mapping of index zero and $N$ is a continuous operator which is $L$-compact on $\bar{\Omega}$.

Now we are in the position of searching for an appropriate open, bounded subset $\Omega$ for the application of the continuation theorem. Corresponding to the operator equation

$$
L u=\lambda N(u, \lambda), \quad \lambda \in(0,1)
$$

we obtain

$$
\begin{aligned}
u_{i}^{\prime}(t)= & \lambda\left[r_{i}(t)-a_{i i}(t) e^{\alpha_{i i} u_{i}(t)}-\lambda \sum_{k=1, k \neq i}^{n} a_{i k}(t) e^{\alpha_{i k} u_{k}(t)}-\lambda \sum_{l=1}^{m} b_{i l}(t) e^{\beta_{i l} u_{n+l}(t)}\right. \\
& \left.-\lambda \sum_{k=1}^{n} c_{i k}(t) e^{\gamma_{i k} u_{k}\left(t-\tau_{i k}(t)\right)}-\lambda \sum_{l=1}^{m} d_{i l}(t) e^{\delta_{i l} u_{n+l}\left(t-\sigma_{i l}(t)\right)}\right], \quad i=1,2, \ldots, n,
\end{aligned}
$$

and

$$
\begin{aligned}
u_{n+j}^{\prime}(t)= & \lambda\left[-\hat{r}_{j}(t)-\hat{b}_{j j}(t) e^{\hat{\beta}_{j j} u_{n+j}(t)}+\sum_{k=1}^{n} \hat{a}_{k j}(t) e^{\hat{\alpha}_{k j} u_{k}(t)}-\lambda \sum_{l=1, l \neq j}^{m} \hat{b}_{l j}(t) e^{\hat{\beta}_{l j} u_{n+l}(t)}\right. \\
& \left.+\sum_{k=1}^{n} \hat{c}_{k j}(t) e^{\hat{\gamma}_{k j} u_{k}\left(t-\hat{\tau}_{k j}(t)\right)}-\lambda \sum_{l=1}^{m} \hat{d}_{l j}(t) e^{\hat{\delta}_{l j} u_{n+l}\left(t-\hat{\sigma}_{l j}(t)\right)}\right], \quad j=1,2, \ldots, m .
\end{aligned}
$$

Assume that $u \in X$ is an almost periodic solution of (3.4)-(3.5), for some $\lambda \in(0,1)$. Then, by Lemma 2.6, for any $\epsilon>0$, there exist $\xi_{h}, \eta_{h} \in \mathbb{R}$ such that $u_{h}\left(\xi_{h}\right)>u_{h}^{M}-\epsilon, u_{h}\left(\eta_{h}\right)<u_{h}^{l}+\epsilon$ and $u_{h}^{\prime}\left(\xi_{h}\right)=u_{h}^{\prime}\left(\eta_{h}\right)=0, h=1,2, \ldots, n+m$. From this and (3.4)-(3.5), for $i=1,2, \ldots, n, j=$ 
$1,2, \ldots, m$, we have

$$
\begin{aligned}
& r_{i}\left(\xi_{i}\right)-a_{i i}\left(\xi_{i}\right) e^{\alpha_{i i} u_{i}\left(\xi_{i}\right)}-\lambda \sum_{k=1, k \neq i}^{n} a_{i k}\left(\xi_{i}\right) e^{\alpha_{i k} u_{k}\left(\xi_{i}\right)}-\lambda \sum_{l=1}^{m} b_{i l}\left(\xi_{i}\right) e^{\beta_{i l} u_{n+l}\left(\xi_{i}\right)} \\
& -\lambda \sum_{k=1}^{n} c_{i k}\left(\xi_{i}\right) e^{\gamma_{i k} u_{k}\left(\xi_{i}-\tau_{i k}\left(\xi_{i}\right)\right)}-\lambda \sum_{l=1}^{m} d_{i l}\left(\xi_{i}\right) e^{\delta_{i l} u_{n+l}\left(\xi_{i}-\sigma_{i l}\left(\xi_{i}\right)\right)}=0, \\
& -\hat{r}_{j}\left(\xi_{n+j}\right)-\hat{b}_{j j}\left(\xi_{n+j}\right) e^{\hat{\beta}_{j j} u_{n+j}\left(\xi_{n+j}\right)}+\sum_{k=1}^{n} \hat{a}_{k j}\left(\xi_{n+j}\right) e^{\hat{\alpha}_{k j} u_{k}\left(\xi_{n+j}\right)}-\lambda \sum_{l=1, l \neq j}^{m} \hat{b}_{l j}\left(\xi_{n+j}\right) e^{\hat{\beta}_{l j} u_{n+l}\left(\xi_{n+j}\right)} \\
& \quad+\sum_{k=1}^{n} \hat{c}_{k j}\left(\xi_{n+j}\right) e^{\hat{\gamma}_{k j} u_{k}\left(\xi_{n+j}-\hat{\tau}_{k j}\left(\xi_{n+j}\right)\right)}-\lambda \sum_{l=1}^{m} \hat{d}_{l j}\left(\xi_{n+j}\right) e^{\hat{\delta}_{l j} u_{n+l}\left(\xi_{n+j}-\hat{\sigma}_{l j}\left(\xi_{n+j}\right)\right)}=0, \\
& r_{i}\left(\eta_{i}\right)-a_{i i}\left(\eta_{i}\right) e^{\alpha_{i i} u_{i}\left(\eta_{i}\right)}-\lambda \sum_{k=1, k \neq i}^{n} a_{i k}\left(\eta_{i}\right) e^{\alpha_{i k} u_{k}\left(\eta_{i}\right)}-\lambda \sum_{l=1}^{m} b_{i l}\left(\eta_{i}\right) e^{\beta_{i l} u_{n+l}\left(\eta_{i}\right)} \\
& \quad-\lambda \sum_{k=1}^{n} c_{i k}\left(\eta_{i}\right) e^{\gamma_{i k} u_{k}\left(\eta_{i}-\tau_{i k}\left(\eta_{i}\right)\right)}-\lambda \sum_{l=1}^{m} d_{i l}\left(\eta_{i}\right) e^{\delta_{i l} u_{n+l}\left(\eta_{i}-\sigma_{i l}\left(\eta_{i}\right)\right)}=0,
\end{aligned}
$$

and

$$
\begin{aligned}
& -\hat{r}_{j}\left(\eta_{n+j}\right)-\hat{b}_{j j}\left(\eta_{n+j}\right) e^{\hat{\beta}_{j j} u_{n+j}\left(\eta_{n+j}\right)}+\sum_{k=1}^{n} \hat{a}_{k j}\left(\eta_{n+j}\right) e^{\hat{\alpha}_{k j} u_{k}\left(\eta_{n+j}\right)}-\lambda \sum_{l=1, l \neq j}^{m} \hat{b}_{l j}\left(\eta_{n+j}\right) e^{\hat{\beta}_{l j} u_{n+l}\left(\eta_{n+j}\right)} \\
& +\sum_{k=1}^{n} \hat{c}_{k j}\left(\eta_{n+j}\right) e^{\hat{\gamma}_{k j} u_{k}\left(\eta_{n+j}-\hat{\tau}_{k j}\left(\eta_{n+j}\right)\right)}-\lambda \sum_{l=1}^{m} \hat{d}_{l j}\left(\eta_{n+j}\right) e^{\hat{\delta}_{l j} u_{n+l}\left(\eta_{n+j}-\hat{\sigma}_{l j}\left(\eta_{n+j}\right)\right)}=0
\end{aligned}
$$

On the one hand, according to (3.6), we have

$$
\begin{aligned}
a_{i i}^{l} e^{\alpha_{i i} u_{i}\left(\xi_{i}\right)} \leq & a_{i i}\left(\xi_{i}\right) e^{\alpha_{i i} u_{i}\left(\xi_{i}\right)} \\
< & a_{i i}\left(\xi_{i}\right) e^{\alpha_{i i} u_{i}\left(\xi_{i}\right)}+\lambda \sum_{k=1, k \neq i}^{n} a_{i k}\left(\xi_{i}\right) e^{\alpha_{i k} u_{k}\left(\xi_{i}\right)}+\lambda \sum_{l=1}^{m} b_{i l}\left(\xi_{i}\right) e^{\beta_{i l} u_{n+l}\left(\xi_{i}\right)} \\
& +\lambda \sum_{k=1}^{n} c_{i k}\left(\xi_{i}\right) e^{\gamma_{i k} u_{k}\left(\xi_{i}-\tau_{i k}\left(\xi_{i}\right)\right)}+\lambda \sum_{l=1}^{m} d_{i l}\left(\xi_{i}\right) e^{\delta_{i l} u_{n+l}\left(\xi_{i}-\sigma_{i l}\left(\xi_{i}\right)\right)} \\
= & r_{i}\left(\xi_{i}\right) \leq r_{i}^{M}
\end{aligned}
$$

which implies that

$$
u_{i}\left(\xi_{i}\right)<\ln l_{i}^{+}, \quad i=1,2, \ldots, n .
$$

By means of (3.7), we obtain

$$
\begin{aligned}
\hat{b}_{j j}^{l} e^{\hat{\beta}_{j j} u_{n+j}\left(\xi_{n+j}\right)} \leq & \hat{b}_{j j}\left(\xi_{n+j}\right) e^{\hat{\beta}_{j j} u_{n+j}\left(\xi_{n+j}\right)} \\
< & \hat{r}_{j}\left(\xi_{n+j}\right)+\hat{b}_{j j}\left(\xi_{n+j}\right) e^{\hat{\beta}_{i j} u_{n+j}\left(\xi_{n+j}\right)} \\
& +\lambda \sum_{l=1, l \neq j}^{m} \hat{b}_{l j}\left(\xi_{n+j}\right) e^{\hat{\beta}_{l j} u_{n+l}\left(\xi_{n+j}\right)}+\lambda \sum_{l=1}^{m} \hat{d}_{l j}\left(\xi_{n+j}\right) e^{\hat{\delta}_{l j} u_{n+l}\left(\xi_{n+j}-\hat{\sigma}_{l j}\left(\xi_{n+j}\right)\right)}
\end{aligned}
$$




$$
\begin{aligned}
& =\sum_{k=1}^{n} \hat{a}_{k j}\left(\xi_{n+j}\right) e^{\hat{\alpha}_{k j} u_{k}\left(\xi_{n+j}\right)}+\sum_{k=1}^{n} \hat{c}_{k j}\left(\xi_{n+j}\right) e^{\hat{\gamma}_{k j} u_{k}\left(\xi_{n+j}-\hat{t}_{k j}\left(\xi_{n+j}\right)\right)} \\
& \leq \sum_{k=1}^{n} \hat{a}_{k j}^{M}\left(l_{k}^{+}\right)^{\hat{\alpha}_{k j}}+\sum_{k=1}^{n} \hat{c}_{k j}^{M}\left(l_{k}^{+}\right)^{\hat{\gamma}_{k j}}
\end{aligned}
$$

which indicates that

$$
u_{n+j}\left(\xi_{n+j}\right)<\ln l_{n+j}^{+}, \quad j=1,2, \ldots, m .
$$

Similar to (3.10)-(3.13), it follows from (3.8) and (3.9) that

$$
u_{i}\left(\eta_{i}\right)<\ln l_{i}^{+}, \quad i=1,2, \ldots, n,
$$

and

$$
u_{n+j}\left(\eta_{n+j}\right)<\ln l_{n+j}^{+}, \quad j=1,2, \ldots, m .
$$

On the other hand, in view of (3.8), we obtain

$$
\begin{aligned}
r_{i}^{l} \leq & r_{i}\left(\eta_{i}\right)=a_{i i}\left(\eta_{i}\right) e^{\alpha_{i i} u_{i}\left(\eta_{i}\right)}+\lambda \sum_{k=1, k \neq i}^{n} a_{i k}\left(\eta_{i}\right) e^{\alpha_{i k} u_{k}\left(\eta_{i}\right)}+\lambda \sum_{l=1}^{m} b_{i l}\left(\eta_{i}\right) e^{\beta_{i l} u_{n+l}\left(\eta_{i}\right)} \\
& +\lambda \sum_{k=1}^{n} c_{i k}\left(\eta_{i}\right) e^{\gamma_{i k} u_{k}\left(\eta_{i}-\tau_{i k}\left(\eta_{i}\right)\right)}+\lambda \sum_{l=1}^{m} d_{i l}\left(\eta_{i}\right) e^{\delta_{i l} u_{n+l}\left(\eta_{i}-\sigma_{i l}\left(\eta_{i}\right)\right)} \\
\leq & a_{i i}^{M} e^{\alpha_{i i} u_{i}\left(\eta_{i}\right)}+\sum_{k=1, k \neq i}^{n} a_{i k}^{M}\left(l_{k}^{+}\right)^{\alpha_{i k}}+\sum_{l=1}^{m} b_{i l}^{M}\left(l_{n+l}^{+}\right)^{\beta_{i l}} \\
& +\sum_{k=1}^{n} c_{i k}^{M}\left(l_{k}^{+}\right)^{\gamma_{i k}}+\sum_{l=1}^{m} d_{i l}^{M}\left(l_{n+l}^{+}\right)^{\delta_{i l}}
\end{aligned}
$$

which implies that

$$
u_{i}\left(\eta_{i}\right) \geq \ln l_{i}^{-}, \quad i=1,2, \ldots, n .
$$

According to (3.9), we get

$$
\begin{aligned}
& \sum_{k=1}^{n} \hat{a}_{k j}^{l}\left(l_{k}^{-}\right)^{\hat{\alpha}_{k j}}+\sum_{k=1}^{n} \hat{c}_{k j}^{l}\left(l_{k}^{-}\right)^{\hat{\gamma}_{k j}} \\
& \leq \sum_{k=1}^{n} \hat{a}_{k j}\left(\eta_{n+j}\right) e^{\hat{\alpha}_{k j} u_{k}\left(\eta_{n+j}\right)}+\sum_{k=1}^{n} \hat{c}_{k j}\left(\eta_{n+j}\right) \hat{\gamma}^{\hat{\gamma}_{k j} u_{k}\left(\eta_{n+j}-\hat{\tau}_{k j}\left(\eta_{n+j}\right)\right)} \\
& =\hat{r}_{j}\left(\eta_{n+j}\right)+\hat{b}_{j j}\left(\eta_{n+j}\right) e^{\hat{\beta}_{j j} u_{n+j}\left(\eta_{n+j}\right)}+\lambda \sum_{l=1, l \neq j}^{m} \hat{b}_{l j}\left(\eta_{n+j}\right) e^{\hat{\beta}_{l j} u_{n+l}\left(\eta_{n+j}\right)} \\
& \quad+\lambda \sum_{l=1}^{m} \hat{d}_{l j}\left(\eta_{n+j}\right) e^{\hat{\delta}_{l j} u_{n+l}\left(\eta_{n+j}-\hat{\sigma}_{l j}\left(\eta_{n+j}\right)\right)} \\
& \leq \hat{r}_{j}^{M}+\hat{b}_{j j}^{M} e^{\hat{\beta}_{j j} u_{n+j}\left(\eta_{n+j}\right)}+\sum_{l=1, l \neq j}^{m} \hat{b}_{l j}^{M}\left(l_{n+l}^{+}\right)^{\hat{\beta}_{l j}}+\sum_{l=1}^{m} \hat{d}_{l j}^{M}\left(l_{n+l}^{+}\right)^{\hat{\delta}_{l j}}
\end{aligned}
$$


which implies that

$$
u_{n+j}\left(\eta_{n+j}\right) \geq \ln l_{n+j}^{-}, \quad j=1,2, \ldots, m .
$$

Similar to (3.16)-(3.19), it follows from (3.6) and (3.7) that

$$
u_{i}\left(\eta_{i}\right) \geq \ln l_{i}^{-}, \quad i=1,2, \ldots, n
$$

and

$$
u_{n+j}\left(\eta_{n+j}\right) \geq \ln l_{n+j}^{-}, \quad j=1,2, \ldots, m
$$

By (3.11), (3.13)-(3.15), (3.17), (3.19)-(3.21) and noticing that $0<l_{h}^{-}<l_{h}^{+}(h=1,2, \ldots, n+$ $m)$, we have, for all $t \in \mathbb{R}$,

$$
\ln l_{h}^{-} \leq u_{h}(t)<\ln l_{h}^{+}, \quad h=1,2, \ldots, n+m .
$$

Denote the open bounded set $\Omega \subset X$ by $\Omega=I_{1} \times I_{2} \times \cdots \times I_{n+m}$, where $I_{h}=\left(\ln l_{h}^{-}-\right.$ $\left.\left.\theta_{h}\right), \ln l_{h}^{+}+\theta_{h}\right), \theta_{h} \in(0, \infty)(h=1,2, \ldots, n+m)$. Clearly, $l_{h}^{+}, l_{h}^{-}$and $\theta_{h}(h=1,2, \ldots, n+m)$ are independent of $\lambda$. Thus $\Omega$ satisfies the requirement (a) in Lemma 3.1.

Now we show that (b) of Lemma 3.1 holds, namely, we prove when $u \in \partial \Omega \cap \operatorname{Ker}(L)=$ $\partial \Omega \cap \mathbb{R}^{n+m}, Q N(u, 0) \neq(0,0, \ldots, 0)^{T}$. If it is not true, then when $u \in \partial \Omega \cap \operatorname{Ker}(L)=\partial \Omega \cap$ $\mathbb{R}^{n+m}$, constant vector $u=\left(u_{1}, u_{2}, \ldots, u_{n+m}\right)^{T}$ with $u \in \partial \Omega$ satisfies

$$
\begin{aligned}
& m\left(r_{i}(t)-\sum_{k=1}^{n} a_{i k}(t) e^{\alpha_{i k} u_{k}}-\sum_{l=1}^{m} b_{i l}(t) e^{\beta_{i l} u_{n+l}}-\sum_{k=1}^{n} c_{i k}(t) e^{\gamma_{i k} u_{k}}-\sum_{l=1}^{m} d_{i l}(t) e^{\delta_{i l} u_{n+l}}\right)=0, \\
& m\left(-\hat{r}_{j}(t)+\sum_{k=1}^{n} \hat{a}_{k j}(t) e^{\hat{\alpha}_{k j} u_{k}}-\sum_{l=1}^{m} \hat{b}_{l j}(t) e^{\hat{\beta}_{l j} u_{n+l}}+\sum_{k=1}^{n} \hat{c}_{k j}(t) e^{\hat{\gamma}_{k j} u_{k}}-\sum_{l=1}^{m} \hat{d}_{l j}(t) e^{\hat{\delta}_{l j} u_{n+l}}\right)=0 .
\end{aligned}
$$

In view of the mean value theorem of calculous, there exist $n+m$ points $t_{i}, \hat{t}_{j}(i=1,2, \ldots, n$; $j=1,2, \ldots, m)$ such that

$$
\begin{aligned}
& r_{i}\left(t_{i}\right)-\sum_{k=1}^{n} a_{i k}\left(t_{i}\right) e^{\alpha_{i k} u_{k}}-\sum_{l=1}^{m} b_{i l}\left(t_{i}\right) e^{\beta_{i l} u_{n+l}}-\sum_{k=1}^{n} c_{i k}\left(t_{i}\right) e^{\gamma_{i k} u_{k}}-\sum_{l=1}^{m} d_{i l}\left(t_{i}\right) e^{\delta_{i l} u_{n+l}} \\
& \quad=0 \\
& -\hat{r}_{j}\left(\hat{t}_{j}\right)+\sum_{k=1}^{n} \hat{a}_{k j}\left(\hat{t}_{j}\right) e^{\hat{\alpha}_{k j} u_{k}}-\sum_{l=1}^{m} \hat{b}_{l j}\left(\hat{t}_{j}\right) e^{\hat{\beta}_{l j} u_{n+l}}+\sum_{k=1}^{n} \hat{c}_{k j}\left(\hat{t}_{j}\right) e^{\hat{\gamma}_{k j} u_{k}}-\sum_{l=1}^{m} \hat{d}_{l j}\left(\hat{t}_{j}\right) e^{\hat{\delta}_{l j} u_{n+l}} \\
& \quad=0 .
\end{aligned}
$$

By the analogous argument of (3.10)-(3.21), it follows from (3.23)-(3.24) that $\ln l_{h}^{-}-\theta_{h}<$ $u_{h}<\ln l_{h}^{+}+\theta_{h}(h=1,2, \ldots, n+m)$, that is, $u \in \Omega \cap \mathbb{R}^{n+m}$. This contradicts the fact that $u \in \partial \Omega \cap \mathbb{R}^{n+m}$. So (b) of Lemma 3.1 holds. 
Next we show that (c) in Lemma 3.1 holds. Consider the following algebraic equation:

$$
\begin{aligned}
r_{i}(t) & -a_{i i}(t) e^{\alpha_{i i} u_{i}}-\sum_{k=1, k \neq i}^{n} a_{i k}(t)\left(l_{k}^{+}\right)^{\alpha_{i k}}-\sum_{l=1}^{m} b_{i l}(t)\left(l_{n+j}^{+}\right)^{\beta_{i l}} \\
& -\sum_{k=1}^{n} c_{i k}(t)\left(l_{k}^{+}\right)^{\gamma_{i k}}-\sum_{l=1}^{m} d_{i l}(t)\left(l_{n+j}^{+}\right)^{\delta_{i l}}=0, \quad i=1,2, \ldots, n,
\end{aligned}
$$

and

$$
\begin{aligned}
& -\hat{r}_{j}(t)-\hat{b}_{j j}(t) e^{\hat{\beta}_{j j} u_{n+j}}+\sum_{k=1}^{n} \hat{a}_{k j}(t)\left(l_{k}^{-}\right)^{\hat{\alpha}_{k j}}-\lambda \sum_{l=1, l \neq j}^{m} \hat{b}_{l j}(t)\left(l_{n+l}^{+}\right)^{\hat{\beta}_{l j}} \\
& +\sum_{k=1}^{n} \hat{c}_{k j}(t)\left(l_{k}^{-}\right)^{\hat{\gamma}_{k j}}-\lambda \sum_{l=1}^{m} \hat{d}_{l j}(t)\left(l_{n+l}^{+}\right)^{\hat{\delta}_{l j}}=0, \quad j=1,2, \ldots, m .
\end{aligned}
$$

Obviously, (3.25)-(3.26) has an unique solution $\left(u_{1}^{*}, u_{2}^{*}, \ldots, u_{n+m}^{*}\right)$. It is easy to verify that $\ln l_{h}^{-}-\theta_{h}<u_{h}^{*}<\ln l_{h}^{+}+\theta_{h}(h=1,2, \ldots, n+m)$. Therefore, $\left(u_{1}^{*}, u_{2}^{*}, \ldots, u_{n+m}^{*}\right) \in \Omega$. Since $\operatorname{Ker}(L)=\operatorname{Im}(Q)$, we can take $J=I$. A direct computation gives

$$
\begin{aligned}
& \operatorname{deg}\left\{\operatorname{JQN}(u, 0), \Omega \cap \operatorname{Ker}(L),(0,0, \ldots, 0)^{T}\right\} \\
& =\operatorname{sign}\left[\prod_{i=1}^{n} \prod_{j=1}^{m}\left(-a_{i i}(t) u_{i}^{*}\right)\left(-\hat{b}_{j j}(t) u_{n+j}^{*}\right)\right]= \pm 1 .
\end{aligned}
$$

So far, we have proved that $\Omega$ satisfies all the assumptions in Lemma 3.1. Hence, system (3.1) has at least an almost periodic solution $\left(\bar{u}_{1}(t), \bar{u}_{2}(t), \ldots, \bar{u}_{n+m}(t)\right)$. Therefore, system (1.4) has at least one positive almost periodic solution $\left(e^{\bar{u}_{1}(t)}, e^{\bar{u}_{2}(t)}, \ldots, e^{\bar{u}_{n+m}(t)}\right)$. The proof is complete.

\section{Global exponential stability}

The aim of this section is to derive the sufficient condition of a unique globally exponentially stable positive almost periodic solution of (1.4).

Under the assumption of Theorem 3.1, we know that system (1.4) has at least one positive almost periodic solution $\left(\bar{x}_{1}(t), \bar{x}_{2}(t), \ldots, \bar{x}_{n+m}(t)\right)$ satisfying $l_{h}^{-} \leq \bar{x}_{h}(t)<l_{h}^{+}(h=$ $1,2, \ldots, n+m)$. Now let $\rho$ be a positive constant satisfying $0<\rho<\min _{1 \leq h \leq n+m}\left\{l_{h}^{-}\right\}$. We assume further that

$\left(H_{4}\right) \tau_{i k}(t), \sigma_{i l}(t), \hat{\tau}_{k j}(t), \sigma_{l j}(t)(i, k=1,2, \ldots, n ; j, l=1,2, \ldots, m) \in C^{1}\left(\mathbb{R}, \mathbb{R}_{0}^{+}\right)$satisfy $0 \leq$ $\tau_{i k}^{\prime}(t), \sigma_{i l}^{\prime}(t), \hat{\tau}_{k j}^{\prime}(t) \sigma_{l j}^{\prime}(t)<1(i, k=1,2, \ldots, n ; j, l=1,2, \ldots, m)$.

$\left(H_{5}\right) \alpha_{i i} \geq \max _{1 \leq k \leq n, 1 \leq l \leq m}\left\{\alpha_{i k}, \beta_{i l}, \gamma_{i k}, \delta_{i l}\right\}, \hat{\beta}_{j j} \geq \max _{1 \leq k \leq n, 1 \leq l \leq m}\left\{\hat{\alpha}_{k j}, \hat{\beta}_{l j}, \hat{\gamma}_{k j}, \hat{\delta}_{l j}\right\}, \quad i=1$, $2, \ldots, n, j=1,2, \ldots, m$.

$\left(H_{6}\right)-\alpha_{i i} \rho a_{i i}^{l}+\sum_{k=1, k \neq i}^{n} \alpha_{k k} \rho^{\frac{\alpha_{k i}}{\alpha_{k k}}} a_{k i}^{M}+\sum_{l=1}^{m} \hat{\beta}_{l l} \rho^{\frac{\hat{\alpha}_{l i}}{\hat{\beta}_{l l}}} \hat{a}_{l i}^{M}+\sum_{k=1}^{n} \alpha_{k k} \rho^{\frac{\gamma_{k i}}{\alpha_{k k}}} c_{k i}^{M}+\sum_{l=1}^{m} \hat{\beta}_{l l} \rho^{\frac{\hat{\gamma}}{\hat{\beta}_{l l}}} \hat{c}_{l i}^{M}<$

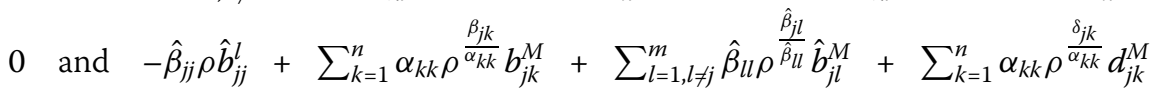
$+\sum_{l=1}^{m} \hat{\beta}_{l l} \rho^{\frac{\hat{\beta}_{j l}}{\hat{\beta}_{l l}}} \hat{d}_{j l}^{M}<0, i=1,2, \ldots, n ; j=1,2, \ldots, m$. 
Making the change of variable $y_{i}(t)=\frac{1}{\rho} x_{i}^{\alpha_{i i}}(t)(i=1,2, \ldots, n), y_{n+j}(t)=\frac{1}{\rho} x_{n+j}^{\hat{\beta}_{j j}}(t) \quad(j=$ $1,2, \ldots, m)$, then system (1.4) is transformed into

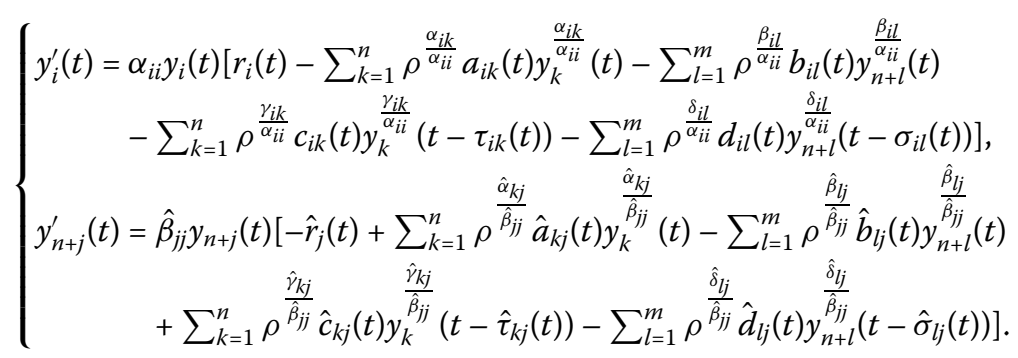

Obviously, $\bar{y}(t)=\left(\bar{y}_{1}(t), \ldots, \bar{y}_{n}(t), \bar{y}_{n+1}(t), \ldots, \bar{y}_{n+m}(t)\right)^{T}$ is the positive almost periodic solution of system (4.1), where $\bar{y}_{i}(t)=\frac{1}{\rho} \bar{x}_{i}^{\alpha i i}(t)(i=1,2, \ldots, n), \bar{y}_{n+j}(t)=\frac{1}{\rho} \bar{x}_{n+j}^{\hat{\beta}_{j j}}(t)(j=1,2, \ldots, m)$. From Theorem 3.1, we know that $l_{h}^{-} \leq \bar{x}_{h}(t)<l_{h}^{+}(h=1,2, \ldots, n+m)$. Therefore,

$$
1<\frac{1}{\rho}\left(l_{i}^{-}\right)^{\alpha_{i i}} \leq \bar{y}_{i}(t)<\frac{1}{\rho}\left(l_{i}^{+}\right)^{\alpha_{i i}}, \quad 1<\frac{1}{\rho}\left(l_{n+j}^{-}\right)^{\hat{\beta}_{i j}} \leq \bar{y}_{n+j}(t)<\frac{1}{\rho}\left(l_{n+j}^{+}\right)^{\hat{\beta}_{j j}} .
$$

Theorem 4.1 Assume that $\left(H_{1}\right)-\left(H_{6}\right)$ hold. Then for system (1.4) there exists a unique positive almost periodic solution which is globally exponentially stable.

Proof According to conditions $\left(H_{1}\right)-\left(H_{3}\right)$, it follows from Theorem 3.1 that system (4.1) has a positive almost periodic solution $\bar{y}(t)=\left(\bar{y}_{1}(t), \bar{y}_{2}(t), \ldots, \bar{y}_{n+m}(t)\right)^{T}$. Let $y(t)=$ $\left(y_{1}(t), y_{2}(t), \ldots, y_{n+m}(t)\right)^{T}$ be any positive solution of system (4.1). Now we construct a Lyapunov functional $V(t)=V_{1}(t)+V_{2}(t)$, where

$$
V_{1}(t)=\sum_{i=1}^{n}\left|\ln y_{i}(t)-\ln \bar{y}_{i}(t)\right|+\sum_{j=1}^{m}\left|\ln y_{n+j}(t)-\ln \bar{y}_{n+j}(t)\right|
$$

and

$$
\begin{aligned}
V_{2}(t)= & \sum_{i=1}^{n} \sum_{k=1}^{n} \alpha_{i i} \rho^{\frac{\gamma_{i k}}{\alpha_{i i}}} c_{i k}^{M} \int_{t-\tau_{i k}(t)}^{t}\left|y_{k}^{\frac{\gamma_{i k}}{\alpha_{i i}}}(s)-\bar{y}_{k}^{\frac{\gamma_{i k}}{\alpha_{i i}}}(s)\right| d s \\
& +\sum_{i=1}^{n} \sum_{l=1}^{m} \alpha_{i i} \rho^{\frac{\delta_{i l}}{\alpha_{i i}}} d_{i l}^{M} \int_{t-\sigma_{i l}(t)}^{t}\left|y_{n+l}^{\frac{\delta_{i l}}{\alpha_{i i}}}(s)-\bar{y}_{n+l}^{\frac{\delta_{i l}}{\alpha_{i i}}}(s)\right| d s \\
& +\sum_{j=1}^{m} \sum_{k=1}^{n} \hat{\beta}_{j j} \rho^{\frac{\hat{\gamma}_{k j}}{\hat{\beta}_{i j}}} \hat{c}_{k j}^{M} \int_{t-\hat{\tau}_{k j}(t)}^{t}\left|y_{k}^{\frac{\hat{\gamma}_{k j}}{\hat{\beta}_{j j}}}(s)-\bar{y}_{k}^{\frac{\hat{\gamma}_{k j}}{\hat{\beta}_{j j}}}(s)\right| d s \\
& +\sum_{j=1}^{m} \sum_{l=1}^{m} \hat{\beta}_{j j} \rho^{\frac{\hat{\delta}_{l j}}{\hat{\beta}_{j j}}} \hat{d}_{l j}^{M} \int_{t-\hat{\sigma}_{l j}(t)}^{t} \mid y_{n+l}^{\frac{\hat{\delta}_{l j}}{\hat{\beta}_{j j}}(s)-\bar{y}_{n+l}^{\hat{\beta}_{l j}}(s) \mid d s .}
\end{aligned}
$$

From the definition of $V(t)$, we easily see that $V(0)<+\infty$ and $V(t) \geq V_{1}(t)$. Noticing that $\operatorname{sgn}\left(\ln y_{h}(t)-\ln \bar{y}_{h}(t)\right)=\operatorname{sgn}\left(y_{h}(t)-\bar{y}_{h}(t)\right)(h=1,2, \ldots, n+m)$, we have

$$
\begin{aligned}
& D^{+}\left(\left|\ln y_{i}(t)-\ln \bar{y}_{i}(t)\right|\right) \\
& \quad=\operatorname{sgn}\left(\ln y_{i}(t)-\ln \bar{y}_{i}(t)\right)\left(\frac{y_{i}^{\prime}(t)}{y_{i}(t)}-\frac{\bar{y}_{i}^{\prime}(t)}{\bar{y}_{i}(t)}\right)
\end{aligned}
$$




$$
\begin{aligned}
& =\operatorname{sgn}\left(y_{i}(t)-\bar{y}_{i}(t)\right)\left(\frac{y_{i}^{\prime}(t)}{y_{i}(t)}-\frac{\bar{y}_{i}^{\prime}(t)}{\bar{y}_{i}(t)}\right) \\
& =\operatorname{sgn}\left(y_{i}(t)-\bar{y}_{i}(t)\right)\left[-\alpha_{i i} \rho a_{i i}(t)\left(y_{i}(t)-\bar{y}_{i}(t)\right)\right. \\
& -\sum_{k=1, k \neq i}^{n} \alpha_{i i} \rho^{\frac{\alpha_{i k}}{\alpha_{i i}}} a_{i k}(t)\left(y_{k}^{\frac{\alpha_{i k}}{\alpha_{i i}}}(t)-\bar{y}_{k}^{\frac{\alpha_{i k}}{\alpha_{i i}}}(t)\right)-\sum_{l=1}^{m} \alpha_{i i} \rho^{\frac{\beta_{i l}}{\alpha_{i i}}} b_{i l}(t)\left(y_{n+l}^{\frac{\beta_{i l}}{\alpha_{i i}}}(t)-\bar{y}_{n+l}^{\frac{\beta_{i l}}{\alpha_{i i}}}(t)\right) \\
& -\sum_{k=1}^{n} \alpha_{i i} \rho^{\frac{\gamma_{i k}}{\alpha_{i i}}} c_{i k}(t)\left(y_{k}^{\frac{\gamma_{i k}}{\alpha_{i i}}}\left(t-\tau_{i k}(t)\right)-\bar{y}_{k}^{\frac{\gamma_{i k}}{\alpha_{i i}}}\left(t-\tau_{i k}(t)\right)\right) \\
& \left.-\sum_{l=1}^{m} \alpha_{i i} \rho^{\frac{\delta_{i l}}{\alpha_{i i}}} d_{i l}(t)\left(y_{n+l}^{\frac{\delta_{i l}}{\alpha_{i i}}}\left(t-\sigma_{i l}(t)\right)-\bar{y}_{n+l}^{\frac{\delta_{i l}}{\alpha_{i i}}}\left(t-\sigma_{i l}(t)\right)\right)\right] \\
& \leq-\alpha_{i i} \rho a_{i i}^{l}\left|y_{i}(t)-\bar{y}_{i}(t)\right|+\sum_{k=1, k \neq i}^{n} \alpha_{i i} \rho^{\frac{\alpha_{i k}}{\alpha_{i i}}} a_{i k}^{M}\left|y_{k}^{\frac{\alpha_{i k}}{\alpha_{i i}}}(t)-\bar{y}_{k}^{\frac{\alpha_{i k}}{\alpha_{i i}}}(t)\right| \\
& +\sum_{l=1}^{m} \alpha_{i i} \rho^{\frac{\beta_{i l}}{\alpha_{i i}}} b_{i l}^{M}\left|y_{n+l}^{\frac{\beta_{i l}}{\alpha_{i i}}}(t)-\bar{y}_{n+l}^{\frac{\beta_{i l}}{\alpha_{i i}}}(t)\right|+\sum_{k=1}^{n} \alpha_{i i} \rho^{\frac{\gamma_{i k}}{\alpha_{i i}}} c_{i k}^{M}\left|y_{k}^{\frac{\gamma_{i k}}{\alpha_{i i}}}\left(t-\tau_{i k}(t)\right)-\bar{y}_{k}^{\frac{\gamma_{i k}}{\alpha_{i i}}}\left(t-\tau_{i k}(t)\right)\right| \\
& +\sum_{l=1}^{m} \alpha_{i i} \rho^{\frac{\delta_{i l}}{\alpha_{i i}}} d_{i l}^{M}\left|y_{n+l}^{\frac{\delta_{i l}}{\alpha_{i i}}}\left(t-\sigma_{i l}(t)\right)-\bar{y}_{n+l}^{\frac{\delta_{i l}}{\alpha_{i i}}}\left(t-\sigma_{i l}(t)\right)\right|, \quad i=1,2, \ldots, n,
\end{aligned}
$$

and

$$
\begin{aligned}
& D^{+}\left(\left|\ln y_{n+j}(t)-\ln \bar{y}_{n+j}(t)\right|\right) \\
& =\operatorname{sgn}\left(\ln y_{n+j}(t)-\ln \bar{y}_{n+j}(t)\right)\left(\frac{y_{n+j}^{\prime}(t)}{y_{n+j}(t)}-\frac{\bar{y}_{n+j}^{\prime}(t)}{\bar{y}_{n+j}(t)}\right) \\
& =\operatorname{sgn}\left(y_{n+j}(t)-\bar{y}_{n+j}(t)\right)\left(\frac{y_{n+j}^{\prime}(t)}{y_{n+j}(t)}-\frac{\bar{y}_{n+j}^{\prime}(t)}{\bar{y}_{n+j}(t)}\right) \\
& =\operatorname{sgn}\left(y_{n+j}(t)-\bar{y}_{n+j}(t)\right) \\
& \times\left[-\hat{\beta}_{j j} \rho \hat{b}_{j j}(t)\left(y_{n+j}(t)-\bar{y}_{n+j}(t)\right)+\sum_{k=1}^{n} \hat{\beta}_{j j} \rho^{\frac{\hat{\alpha}_{k j}}{\hat{\beta}_{j j}}} \hat{a}_{k j}(t)\left(y_{k}^{\frac{\hat{\alpha}_{k j}}{\hat{\beta}_{j j}}}(t)-\bar{y}_{k}^{\frac{\hat{\alpha}_{k j}}{\hat{\beta}_{j j}}}(t)\right)\right. \\
& -\sum_{l=1, l \neq j}^{m} \hat{\beta}_{j j} \rho^{\frac{\hat{\beta}_{l j}}{\hat{\beta}_{j j}}} \hat{b}_{l j}(t)\left(y_{n+l}^{\frac{\hat{\beta}_{l j}}{\hat{\beta}_{j j}}}(t)-\bar{y}_{n+l}^{\frac{\hat{\beta}_{l j}}{\hat{\beta}_{j i j}}}(t)\right) \\
& +\sum_{k=1}^{n} \hat{\beta}_{j j} \rho^{\frac{\hat{\gamma}_{k j}}{\hat{\beta}_{j j}}} \hat{c}_{k j}(t)\left(y_{k}^{\frac{\hat{\gamma}_{k j}}{\hat{\beta}_{j j}}}\left(t-\hat{\tau}_{k j}(t)\right)-\bar{y}_{k}^{\frac{\hat{\gamma}_{k j}}{\hat{\beta}_{j j}}}\left(t-\hat{\tau}_{k j}(t)\right)\right)
\end{aligned}
$$

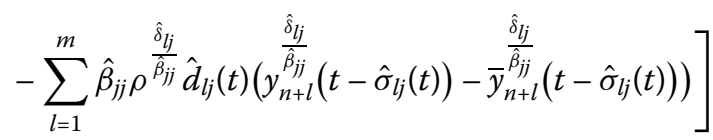

$$
\begin{aligned}
& \leq-\hat{\beta}_{j j} \rho \hat{b}_{j j}^{l}\left|y_{n+j}(t)-\bar{y}_{n+j}(t)\right|+\sum_{k=1}^{n} \hat{\beta}_{j j} \rho^{\frac{\hat{\alpha}_{k j}}{\hat{\beta}_{i j}}} \hat{a}_{k j}^{M}\left|y_{k}^{\frac{\hat{\alpha}_{k j}}{\hat{\beta}_{j j}}}(t)-\bar{y}_{k}^{\frac{\hat{\alpha}_{k j}}{\hat{\beta}_{j j}}}(t)\right|
\end{aligned}
$$




$$
\begin{aligned}
& +\sum_{l=1, l \neq j}^{m} \hat{\beta}_{j j} \rho^{\frac{\hat{\beta}_{l j}}{\hat{\beta}_{j j}}} \hat{b}_{l j}^{M}\left|y_{n+l}^{\hat{\beta}_{l j}}(t)-\bar{y}_{n+l}^{\hat{\beta}_{j j}}(t)\right| \\
& +\sum_{k=1}^{\hat{\beta}_{l j}} \hat{\beta}_{j j} \rho^{\frac{\hat{\gamma}_{k j}}{\hat{\beta}_{j j}}} \hat{c}_{k j}^{M}\left|y_{k}^{\frac{\hat{\gamma}_{k j}}{\hat{\beta}_{j j}}}\left(t-\hat{\tau}_{k j}(t)\right)-\bar{y}_{k}^{\frac{\hat{\gamma}_{k j}}{\hat{\beta}_{i j}}}\left(t-\hat{\tau}_{k j}(t)\right)\right| \\
& +\sum_{l=1}^{m} \hat{\beta}_{j j} \rho^{\frac{\hat{\delta}_{l j}}{\hat{\beta}_{j j}}} \hat{d}_{l j}^{M}\left|y_{n+l}^{\hat{\beta}_{l j}}\left(t-\hat{\sigma}_{l j}(t)\right)-\bar{y}_{n+l}^{\hat{\beta}_{i j}}\left(t-\hat{\sigma}_{l j}(t)\right)\right|, \quad j=1,2, \ldots, m .
\end{aligned}
$$

By the condition $\left(H_{4}\right)$, we obtain

$$
\begin{aligned}
& \left(\int_{t-\tau_{i k}(t)}^{t}\left|y_{k}^{\frac{\gamma_{i k}}{\alpha_{i i}}}(s)-\bar{y}_{k}^{\frac{\gamma_{i k}}{\alpha_{i i}}}(s)\right| d s\right)^{\prime} \\
& =\left|y_{k}^{\frac{\gamma_{i k}}{\alpha_{i i}}}(t)-\bar{y}_{k}^{\frac{\gamma_{i k}}{\alpha_{i i}}}(t)\right|-\left|y_{k}^{\frac{\gamma_{i k}}{\alpha_{i i}}}\left(t-\tau_{i k}(t)\right)-\bar{y}_{k}^{\frac{\gamma_{i k}}{\alpha_{i i}}}\left(t-\tau_{i k}(t)\right)\right|\left(1-\tau_{i k}^{\prime}(t)\right) \\
& \leq\left|y_{k}^{\frac{\gamma_{i k}}{\alpha_{i i}}}(t)-\bar{y}_{k}^{\frac{\gamma_{i k}}{\alpha_{i i}}}(t)\right|-\left|y_{k}^{\frac{\gamma_{i k}}{\alpha_{i i}}}\left(t-\tau_{i k}(t)\right)-\bar{y}_{k}^{\frac{\gamma_{i k}}{\alpha_{i i}}}\left(t-\tau_{i k}(t)\right)\right|, \\
& \left(\int_{t-\sigma_{i l}(t)}^{t}\left|y_{n+l}^{\frac{\delta_{i l}}{\alpha_{i i}}}(s)-\bar{y}_{n+l}^{\frac{\delta_{i l}}{\alpha_{i i}}}(s)\right| d s\right)^{\prime} \\
& =\left|y_{n+l}^{\frac{\delta_{i l}}{\alpha_{i i}}}(t)-\bar{y}_{n+l}^{\frac{\delta_{i l}}{\alpha_{i i}}}(t)\right|-\left|y_{n+l}^{\frac{\delta_{i l}}{\alpha_{i i}}}\left(t-\sigma_{i l}(t)\right)-\bar{y}_{n+l}^{\frac{\delta_{i l}}{\alpha_{i i}}}\left(t-\sigma_{i l}(t)\right)\right|\left(1-\sigma_{i l}^{\prime}(t)\right) \\
& \leq\left|y_{n+l}^{\frac{\delta_{i l}}{\alpha_{i i}}}(t)-\bar{y}_{n+l}^{\frac{\delta_{i l}}{\alpha_{i i}}}(t)\right|-\left|y_{n+l}^{\frac{\delta_{i l}}{\alpha_{i i}}}\left(t-\sigma_{i l}(t)\right)-\bar{y}_{n+l}^{\frac{\delta_{i l}}{\alpha_{i i}}}\left(t-\sigma_{i l}(t)\right)\right|, \\
& \left(\int_{t-\hat{\tau}_{k j}(t)}^{t}\left|y_{k}^{\hat{\beta}_{k j}}(s)-\bar{y}_{k}^{\hat{\beta}_{k j}}(s)\right| d s\right)^{\prime} \\
& =\left|y_{k}^{\hat{\gamma}_{k j}}(t)-\bar{y}_{k}^{\hat{\gamma}_{k j}}(t)\right|-\left|y_{k}^{\frac{\hat{\gamma}_{k j}}{\hat{\beta}_{j i}}}\left(t-\hat{\tau}_{k j}(t)\right)-\bar{y}_{k}^{\frac{\hat{\gamma}_{k j}}{\hat{\beta}_{j j}}}\left(t-\hat{\tau}_{k j}(t)\right)\right|\left(1-\hat{\tau}_{k j}^{\prime}(t)\right) \\
& \leq\left|y_{k}^{\hat{\gamma}_{k j}}(t)-\bar{y}_{k}^{\hat{\beta}_{k j}}(t)\right|-\left|y_{k}^{\hat{\beta}_{k j}}\left(t-\hat{\tau}_{k j}(t)\right)-\bar{y}_{k}^{\hat{\beta}_{k j}}\left(t-\hat{\tau}_{k j}(t)\right)\right|
\end{aligned}
$$

and

$$
\begin{aligned}
& \left(\int_{t-\hat{\sigma}_{l j}(t)}^{t}\left|y_{n+l}^{\frac{\hat{\delta}_{l j}}{\hat{\beta}_{j j}}}(s)-\bar{y}_{n+l}^{\hat{\delta}_{j j}}(s)\right| d s\right)^{\prime}
\end{aligned}
$$

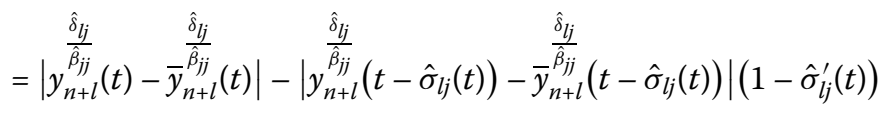

$$
\begin{aligned}
& \leq\left|y_{n+l}^{\hat{\beta}_{l j}}(t)-\bar{y}_{n+l}^{\hat{\beta}_{i j}}(t)\right|-\left|y_{n+l}^{\hat{\beta}_{l j}} \frac{\hat{\delta}_{l j}}{\hat{\beta}_{j j}}\left(t-\hat{\sigma}_{l j}(t)\right)-\bar{y}_{n+l}^{\hat{\beta}_{l j}}\left(t-\hat{\sigma}_{l j}(t)\right)\right| \text {. }
\end{aligned}
$$

By $\left(H_{5}\right)$, we have $0<\frac{\alpha_{i k}}{\alpha_{i i}}, \frac{\beta_{i l}}{\alpha_{i i}}, \frac{\gamma_{i k}}{\alpha_{i i}}, \frac{\delta_{i l}}{\alpha_{i i}}, \frac{\hat{\alpha}_{k j}}{\hat{\beta}_{j j}}, \frac{\hat{\beta}_{l j}}{\hat{\beta}_{j j}}, \frac{\hat{\gamma}_{k j}}{\hat{\beta}_{j j}}, \frac{\hat{\delta}_{l j}}{\hat{\beta}_{j j}} \leq 1$. Observe that $g(x)=\left|a^{x}-b^{x}\right|$ is an increasing function for $a \geq 1$ and $x>0$. Therefore, according to (4.1) and (4.5)-(4.10), we 
derive

$$
\begin{aligned}
& D^{+} V(t) \leq \sum_{i=1}^{n}\left[-\alpha_{i i} \rho a_{i i}^{l}\left|y_{i}(t)-\bar{y}_{i}(t)\right|+\sum_{k=1, k \neq i}^{n} \alpha_{k k} \rho^{\frac{\alpha_{k i}}{\alpha_{k k}}} a_{k i}^{M}\left|y_{i}^{\frac{\alpha_{k i}}{\alpha_{k k}}}(t)-\bar{y}_{i}^{\frac{\alpha_{k i}}{\alpha_{k k}}}(t)\right|\right. \\
& +\sum_{l=1}^{m} \hat{\beta}_{l l} \rho^{\frac{\hat{\alpha}_{l i}}{\hat{\beta}_{l l}}} \hat{a}_{l i}^{M}\left|y_{i}^{\frac{\hat{\alpha}_{l i}}{\hat{\beta}_{l l}}}(t)-\bar{y}_{i}^{\frac{\hat{\alpha}_{l i}}{\hat{\beta}_{l l}}}(t)\right|+\sum_{k=1}^{n} \alpha_{k k} \rho^{\frac{\gamma_{k i}}{\alpha_{k k}}} c_{k i}^{M}\left|y_{i}^{\frac{\gamma_{k i}}{\alpha_{k k}}}(t)-\bar{y}_{i}^{\frac{\gamma_{k i}}{\alpha_{k k}}}(t)\right| \\
& \left.+\sum_{l=1}^{m} \hat{\beta}_{l l} \rho^{\frac{\hat{\gamma}_{l i}}{\hat{\beta}_{l l}}} \hat{c}_{l i}^{M}\left|y_{i}^{\frac{\hat{\gamma}_{l i}}{\hat{\beta}_{l l}}}(t)-\bar{y}_{i}^{\frac{\hat{\beta}_{l i}}{\hat{\beta}_{l l}}}(t)\right|\right]+\sum_{j=1}^{m}\left[-\hat{\beta}_{j j} \rho \hat{b}_{j j}^{l}\left|y_{n+j}(t)-\bar{y}_{n+j}(t)\right|\right. \\
& +\sum_{k=1}^{n} \alpha_{k k} \rho^{\frac{\beta_{j k}}{\alpha_{k k}}} b_{j k}^{M}\left|y_{n+j}^{\frac{\beta_{j k}}{\alpha_{k j}}}(t)-\bar{y}_{n+j}^{\frac{\beta_{j k}}{\alpha_{k k}}}(t)\right|+\sum_{l=1, l \neq j}^{m} \hat{\beta}_{l l} \rho^{\frac{\hat{\beta}_{j l}}{\hat{\beta}_{l l}}} \hat{b}_{j l}^{M}\left|y_{n+j}^{\frac{\hat{\beta}_{j l}}{\hat{\beta}_{l l}}}(t)-\bar{y}_{n+j}^{\frac{\hat{\beta}_{l l}}{\hat{\beta}_{l l}}}(t)\right| \\
& \left.+\sum_{k=1}^{n} \alpha_{k k} \rho^{\frac{\delta_{j k}}{\alpha_{k k}}} d_{j k}^{M}\left|y_{n+j}^{\frac{\delta_{j k}}{\alpha_{k+k}}}(t)-\bar{y}_{n+j}^{\frac{\delta_{j k}}{\alpha_{k k}}}(t)\right|+\sum_{l=1}^{m} \hat{\beta}_{l l} \rho^{\frac{\hat{\delta}_{j l}}{\hat{\beta}_{l l}}} \hat{d}_{j l}^{M}\left|y_{n+j}^{\frac{\hat{\beta}_{j l}}{\hat{\beta}_{l l}}}(t)-\bar{y}_{n+j}^{\frac{\hat{\beta}_{j l}}{\hat{\beta}_{l l}}}(t)\right|\right] \\
& \leq \sum_{i=1}^{n}\left[-\alpha_{i i} \rho a_{i i}^{l}\left|y_{i}(t)-\bar{y}_{i}(t)\right|+\sum_{k=1, k \neq i}^{n} \alpha_{k k} \rho^{\frac{\alpha_{k i}}{\alpha_{k k}}} a_{k i}^{M}\left|y_{i}(t)-\bar{y}_{i}(t)\right|\right. \\
& +\sum_{l=1}^{m} \hat{\beta}_{l l} \rho^{\frac{\hat{\alpha}_{l i}}{\hat{\beta}_{l l}}} \hat{a}_{l i}^{M}\left|y_{i}(t)-\bar{y}_{i}(t)\right|+\sum_{k=1}^{n} \alpha_{k k} \rho^{\frac{\gamma_{k i}}{\alpha_{k k}}} c_{k i}^{M}\left|y_{i}(t)-\bar{y}_{i}(t)\right| \\
& \left.+\sum_{l=1}^{m} \hat{\beta}_{l l} \rho^{\hat{\beta}_{l i}} \hat{c}_{l i}^{M}\left|y_{i}(t)-\bar{y}_{i}(t)\right|\right]+\sum_{j=1}^{m}\left[-\hat{\beta}_{j j} \rho \hat{b}_{j j}^{l}\left|y_{n+j}(t)-\bar{y}_{n+j}(t)\right|\right. \\
& +\sum_{k=1}^{n} \alpha_{k k} \rho^{\frac{\beta_{j k}}{\alpha_{k k}}} b_{j k}^{M}\left|y_{n+j}(t)-\bar{y}_{n+j}(t)\right|+\sum_{l=1, l \neq j}^{m} \hat{\beta}_{l l} \rho^{\frac{\hat{\beta}_{j l}}{\hat{\beta}_{l l}}} \hat{b}_{j l}^{M}\left|y_{n+j}(t)-\bar{y}_{n+j}(t)\right| \\
& \left.+\sum_{k=1}^{n} \alpha_{k k} \rho^{\frac{\delta_{j k}}{\alpha_{k k}}} d_{j k}^{M}\left|y_{n+j}(t)-\bar{y}_{n+j}(t)\right|+\sum_{l=1}^{m} \hat{\beta}_{l l} \rho^{\frac{\hat{\beta}_{j l}}{\hat{\beta}_{l l}}} \hat{d}_{j l}^{M}\left|y_{n+j}(t)-\bar{y}_{n+j}(t)\right|\right] \\
& =\sum_{i=1}^{n}\left[-\alpha_{i i} \rho a_{i i}^{l}+\sum_{k=1, k \neq i}^{n} \alpha_{k k} \rho^{\frac{\alpha_{k i}}{\alpha_{k k}}} a_{k i}^{M}+\sum_{l=1}^{m} \hat{\beta}_{l l} \rho^{\frac{\hat{\alpha}_{\hat{\beta}}}{\hat{\beta}_{l}}} \hat{a}_{l i}^{M}+\sum_{k=1}^{n} \alpha_{k k} \rho^{\frac{\gamma_{k i}}{\alpha_{k k}}} c_{k i}^{M}\right. \\
& \left.+\sum_{l=1}^{m} \hat{\beta}_{l l} \rho^{\frac{\hat{\gamma}_{l i}}{\hat{\beta}_{l l}}} \hat{c}_{l i}^{M}\right]\left|y_{i}(t)-\bar{y}_{i}(t)\right|+\sum_{j=1}^{m}\left[-\hat{\beta}_{j j} \rho \hat{b}_{j j}^{l}+\sum_{k=1}^{n} \alpha_{k k} \rho^{\frac{\beta_{j k}}{\alpha_{k k}}} b_{j k}^{M}\right. \\
& +\sum_{l=1, l \neq j}^{m} \hat{\beta}_{l l} \rho^{\frac{\hat{\beta}_{i l}}{\hat{\beta}_{l l}}} \hat{b}_{j l}^{M}+\sum_{k=1}^{n} \alpha_{k k} \rho^{\frac{\delta_{j k}}{\alpha_{k k}}} d_{j k}^{M} \\
& \left.+\sum_{l=1}^{m} \hat{\beta}_{l l} \rho^{\frac{\hat{\delta}_{j l}}{\hat{\beta}_{l l}}} \hat{d}_{j l}^{M}\right]\left|y_{n+j}(t)-\bar{y}_{n+j}(t)\right| .
\end{aligned}
$$

In addition, from the condition $\left(H_{6}\right)$, there exists a positive constant $\kappa$ such that

$$
-\alpha_{i i} \rho a_{i i}^{l}+\sum_{k=1, k \neq i}^{n} \alpha_{k k} \rho^{\frac{\alpha_{k i}}{\alpha_{k k}}} a_{k i}^{M}+\sum_{l=1}^{m} \hat{\beta}_{l l} \rho^{\frac{\hat{\alpha}_{l i}}{\hat{\beta}_{l l}}} \hat{a}_{l i}^{M}+\sum_{k=1}^{n} \alpha_{k k} \rho^{\frac{\gamma_{k i}}{\alpha_{k k}}} c_{k i}^{M}+\sum_{l=1}^{m} \hat{\beta}_{l l} \rho^{\frac{\hat{\gamma}_{l i}}{\hat{\beta}_{l l}}} \hat{c}_{l i}^{M}+\kappa<0
$$


and

$$
-\hat{\beta}_{j j} \rho \hat{b}_{j j}^{l}+\sum_{k=1}^{n} \alpha_{k k} \rho^{\frac{\beta_{j k}}{\alpha_{k k}}} b_{j k}^{M}+\sum_{l=1, l \neq j}^{m} \hat{\beta}_{l l} \rho^{\frac{\hat{\beta}_{j l}}{\hat{\beta}_{l l}}} \hat{b}_{j l}^{M}+\sum_{k=1}^{n} \alpha_{k k} \rho^{\frac{\delta_{j k}}{\alpha_{k k}}} d_{j k}^{M}+\sum_{l=1}^{m} \hat{\beta}_{l l} \rho^{\frac{\hat{\delta}_{j l}}{\hat{\beta}_{l l}}} \hat{d}_{j l}^{M}+\kappa<0
$$

The above two inequalities together with (4.11) lead to

$$
D^{+} V(t) \leq-\kappa \sum_{h=1}^{n+m}\left|y_{h}(t)-\bar{y}_{h}(t)\right|
$$

Integrating both sides of (4.12) with respect to $t$, we have

$$
V(t)+\kappa \int_{0}^{t} \sum_{h=1}^{n+m}\left|y_{h}(s)-\bar{y}_{h}(s)\right| d s \leq V(0)<+\infty, \quad t \geq 0 .
$$

Equation (4.13) shows that

$$
\int_{0}^{t} \sum_{h=1}^{n+m}\left|y_{h}(s)-\bar{y}_{h}(s)\right| d s \leq \frac{V(0)}{\kappa}<+\infty, \quad t \geq 0
$$

which implies that

$$
\sum_{h=1}^{n+m}\left|y_{h}(s)-\bar{y}_{h}(s)\right| \in L^{1}[0,+\infty)
$$

(4.2) means that $\bar{y}_{h}(t)(h=1,2, \ldots, n+m)$ is uniformly bounded from below and above, and so $\ln \bar{y}_{h}(t)$ is bounded. From $\left|\ln y_{h}(t)-\ln \bar{y}_{h}(t)\right| \leq V_{1}(t) \leq V(t) \leq V(0)$, we get

$$
\bar{y}_{h}(t) e^{-V(0)} \leq y_{h}(t) \leq \bar{y}_{h}(t) e^{V(0)}
$$

(4.16) and (4.2) show that $y_{h}(t), \bar{y}_{h}(t), h=1,2, \ldots, n+m$ are uniformly bounded. This fact together with (4.1) lead to $y_{h}^{\prime}(t), \bar{y}_{h}^{\prime}(t), h=1,2, \ldots, n+m$ are uniformly bounded on $[0,+\infty)$. Therefore $\sum_{h=1}^{n+m}\left|y_{h}(s)-\bar{y}_{h}(s)\right|$ is uniformly continuous on $[0,+\infty)$. From (4.14) we know that $\sum_{h=1}^{n+m}\left|y_{h}(s)-\bar{y}_{h}(s)\right|$ is integrable on $[0,+\infty)$. By Barbalat's lemma (Lemma 1.2.2 and Lemma 1.2.3, [20]), we can conclude that

$$
\lim _{t \rightarrow+\infty}\left|y_{h}(t)-\bar{y}_{h}(t)\right|=0, \quad h=1,2, \ldots, n+m
$$

Thus, we have proved that the positive almost periodic solution $\left(\bar{y}_{1}(t), \bar{y}_{2}(t), \ldots, \bar{y}_{n+m}(t)\right)^{T}$ of system (4.1) is globally attractive.

Next, we shall prove that the positive almost periodic solution $\left(\bar{y}_{1}(t), \bar{y}_{2}(t), \ldots, \bar{y}_{n+m}(t)\right)^{T}$ of system (4.1) is globally exponentially stable. In fact, in the light of (4.2) and (4.17), we know that, for any $\varepsilon>0$ ( $\varepsilon$ enough small), there exists $T>0$ such that for $t>T$

$$
1<\frac{1}{\rho}\left(l_{i}^{-}\right)^{\alpha_{i i}}-\varepsilon \leq \bar{y}_{i}(t)-\varepsilon<y_{i}(t)<\bar{y}_{i}(t)+\varepsilon<\frac{1}{\rho}\left(l_{i}^{+}\right)^{\alpha_{i i}}+\varepsilon
$$


and

$$
1<\frac{1}{\rho}\left(l_{n+j}^{-}\right)^{\hat{\beta}_{j j}}-\varepsilon \leq \bar{y}_{n+j}(t)-\varepsilon<y_{n+j}(t)<\bar{y}_{n+j}(t)+\varepsilon<\frac{1}{\rho}\left(l_{n+j}^{+}\right)^{\hat{\beta}_{j j}}+\varepsilon .
$$

By (4.18)-(4.19) and the mean value theorem of calculus, we have

$$
\left|\ln y_{i}(t)-\ln \bar{y}_{i}(t)\right|=\left|\frac{1}{A_{i}}\right|\left|y_{i}(t)-\bar{y}_{i}(t)\right| \leq \frac{\left|y_{i}(t)-\bar{y}_{i}(t)\right|}{\frac{1}{\rho}\left(l_{i}^{-}\right)^{\alpha_{i i}}-\varepsilon}
$$

and

$$
\left|\ln y_{n+j}(t)-\ln \bar{y}_{n+j}(t)\right|=\left|\frac{1}{A_{n+j}}\right|\left|y_{n+j}(t)-\bar{y}_{n+j}(t)\right| \leq \frac{\left|y_{n+j}(t)-\bar{y}_{n+j}(t)\right|}{\frac{1}{\rho}\left(l_{n+j}^{-}\right)^{\hat{\beta}_{j j}}-\varepsilon},
$$

where $i=1,2, \ldots, n, j=1,2, \ldots, m, A_{h}(h=1,2, \ldots, n+m)$ lies between $y_{h}(t)$ and $\bar{y}_{h}(t)$. According to $\left(H_{6}\right)$, there exists a constant $\mu>0$ such that

$$
\begin{gathered}
-\alpha_{i i} \rho a_{i i}^{l}+\sum_{k=1, k \neq i}^{n} \alpha_{k k} \rho^{\frac{\alpha_{k i}}{\alpha_{k k}}} a_{k i}^{M}+\sum_{l=1}^{m} \hat{\beta}_{l l} \rho^{\frac{\hat{\alpha}_{l i}}{\hat{\beta}_{l l}}} \hat{a}_{l i}^{M}+\sum_{k=1}^{n} \alpha_{k k} \rho^{\frac{\gamma_{k i}}{\alpha_{k k}}} c_{k i}^{M} \\
+\sum_{l=1}^{m} \hat{\beta}_{l l} \rho^{\frac{\hat{\gamma}_{l i}}{\hat{\beta}_{l l}}} \hat{c}_{l i}^{M}+\frac{\mu}{\frac{1}{\rho}\left(l_{i}^{-}\right)^{\alpha_{i i}}-\varepsilon}<0, \quad i=1,2, \ldots, n,
\end{gathered}
$$

and

$$
\begin{gathered}
-\hat{\beta}_{j j} \rho \hat{b}_{j j}^{l}+\sum_{k=1}^{n} \alpha_{k k} \rho^{\frac{\beta_{j k}}{\alpha_{k k}}} b_{j k}^{M}+\sum_{l=1, l \neq j}^{m} \hat{\beta}_{l l} \rho^{\frac{\hat{\beta}_{j l}}{\hat{\beta}_{l l}}} \hat{b}_{j l}^{M}+\sum_{k=1}^{n} \alpha_{k k} \rho^{\frac{\delta_{j k}}{\alpha_{k k}}} d_{j k}^{M} \\
+\sum_{l=1}^{m} \hat{\beta}_{l l} \rho^{\frac{\hat{\beta}_{j l}}{\hat{\beta}_{l l}}} \hat{d}_{j l}^{M}+\frac{\mu}{\frac{1}{\rho}\left(l_{n+j}^{-}\right)^{\hat{\beta}_{j j}}-\varepsilon}<0, \quad j=1,2, \ldots, m .
\end{gathered}
$$

Construct the Lyapunov functional $W(t)=e^{\mu t} V_{1}(t)+V_{2}(t)$. Applying (4.11), (4.22) and (4.23), we have

$$
\begin{aligned}
D^{+} W(t) \leq & \sum_{i=1}^{n}\left[-\alpha_{i i} \rho a_{i i}^{l}+\sum_{k=1, k \neq i}^{n} \alpha_{k k} \rho^{\frac{\alpha_{k i}}{\alpha_{k k}}} a_{k i}^{M}+\sum_{l=1}^{m} \hat{\beta}_{l l} \rho^{\frac{\hat{\alpha}_{l i}}{\hat{\beta}_{l l}}} \hat{a}_{l i}^{M}+\sum_{k=1}^{n} \alpha_{k k} \rho^{\frac{\gamma k i}{\alpha_{k k}}} c_{k i}^{M}\right. \\
& \left.+\sum_{l=1}^{m} \hat{\beta}_{l l} \rho^{\frac{\hat{\gamma}_{l i}}{\hat{\beta}_{l l}}} \hat{c}_{l i}^{M}+\frac{\mu}{\frac{1}{\rho}\left(l_{i}^{-}\right)^{\alpha_{i i}}-\varepsilon}\right]\left|y_{i}(t)-\bar{y}_{i}(t)\right| \\
& +\sum_{j=1}^{m}\left[-\hat{\beta}_{j j} \hat{b}_{j j}^{l}+\sum_{k=1}^{n} \alpha_{k k} \rho^{\frac{\beta_{j k}}{\alpha_{k k}}} b_{j k}^{M}+\sum_{l=1, l \neq j}^{m} \hat{\beta}_{l l} \rho^{\frac{\hat{\beta}_{j l}}{\hat{\beta}_{l}}} \hat{b}_{j l}^{M}+\sum_{k=1}^{n} \alpha_{k k} \rho^{\frac{\delta_{j k}}{\alpha_{k k}}} d_{j k}^{M}\right. \\
& \left.+\sum_{l=1}^{m} \hat{\beta}_{l l} \rho^{\frac{\hat{j}_{l l}}{\hat{\beta}_{l l}}} \hat{d}_{j l}^{M}+\frac{\mu}{\frac{1}{\rho}\left(l_{n+j}^{-}\right)^{\hat{\beta}_{j j}}-\varepsilon}\right]\left|y_{n+j}(t)-\bar{y}_{n+j}(t)\right|<0 .
\end{aligned}
$$

Equation (4.24) means that

$$
W(t)<W(T)=e^{\mu T} V_{1}(T)+V_{2}(T) \leq e^{\mu T} V(T) \leq e^{\mu T} V(0), \quad t>0 .
$$


Similar to (4.18)-(4.21), we also have

$$
\begin{aligned}
& \left|\ln y_{i}(t)-\ln \bar{y}_{i}(t)\right|>\frac{\left|y_{i}(t)-\bar{y}_{i}(t)\right|}{\frac{1}{\rho}\left(l_{i}^{+}\right)^{\alpha_{i i}}+\varepsilon}, \\
& \left|\ln y_{n+j}(t)-\ln \bar{y}_{n+j}(t)\right|>\frac{\left|y_{n+j}(t)-\bar{y}_{n+j}(t)\right|}{\frac{1}{\rho}\left(l_{n+j}^{+}\right)^{\hat{\beta}_{j j}}+\varepsilon} .
\end{aligned}
$$

(4.25) and (4.26) lead to

$$
\frac{e^{\mu t}}{\frac{1}{\rho}\left(l_{i}^{+}\right)^{\alpha_{i i}}+\varepsilon}\left|y_{i}(t)-\bar{y}_{i}(t)\right| \leq e^{\mu t} \sum_{i=1}^{n} \frac{\left|y_{i}(t)-\bar{y}_{i}(t)\right|}{\frac{1}{\rho}\left(l_{i}^{+}\right)^{\alpha_{i i}}+\varepsilon}<e^{\mu t} V_{1}(t) \leq W(t)<e^{\mu T} V(0)
$$

and

$$
\frac{e^{\mu t}}{\frac{1}{\rho}\left(l_{n+j}^{+} \hat{\beta}^{\hat{\beta}_{j j}}+\varepsilon\right.}\left|y_{n+j}(t)-\bar{y}_{n+j}(t)\right| \leq e^{\mu t} \sum_{j=1}^{m} \frac{\left|y_{n+j}(t)-\bar{y}_{n+j}(t)\right|}{\frac{1}{\rho}\left(l_{n+j}^{+}\right)^{\hat{\beta}_{j j}}+\varepsilon}<e^{\mu t} V_{1}(t) \leq W(t)<e^{\mu T} V(0) .
$$

Letting $\varepsilon \rightarrow 0^{+}$in the above two inequalities, we have

$$
\left|y_{h}(t)-\bar{y}_{h}(t)\right|<M e^{-\mu t}, \quad h=1,2, \ldots, n+m, t>0,
$$

where $M=e^{\mu T} V(0) \max _{1 \leq i \leq n, 1 \leq j \leq m}\left\{\frac{1}{\rho}\left(l_{i}^{+}\right)^{\alpha_{i i}}, \frac{1}{\rho}\left(l_{n+j}^{+}\right)^{\hat{\beta}_{j i}}\right\}$. (4.27) indicates that the unique positive almost periodic solution $\left(\bar{y}_{1}(t), \bar{y}_{2}(t), \ldots, \bar{y}_{n+m}(t)\right)^{T}$ of system (4.1) is globally exponentially stable.

Now let us to show that the unique positive almost periodic solution $\left(\bar{x}_{1}(t), \bar{x}_{2}(t), \ldots\right.$, $\left.\bar{x}_{n+m}(t)\right)^{T}$ of system (1.4) is globally exponentially stable.

Indeed, by the mean value theorem of calculus and (4.18)-(4.19), we have

$$
\left|x_{i}(t)-\bar{x}_{i}(t)\right|=\rho^{\frac{1}{\alpha_{i i}}}\left|y_{i}^{\frac{1}{\alpha_{i i}}}(t)-\bar{y}_{i}^{\frac{1}{\alpha_{i i}}}(t)\right| \leq \frac{N_{i}(\varepsilon)}{\alpha_{i i}} \rho^{\frac{1}{\alpha_{i i}}}\left|y_{i}(t)-\bar{y}_{i}(t)\right|
$$

and

$$
\left|x_{n+j}(t)-\bar{x}_{n+j}(t)\right|=\rho^{\frac{1}{\hat{\beta}_{j j}}}\left|y_{n+j}^{\frac{1}{\hat{\beta}_{j i}}}(t)-\bar{y}_{n+j}^{\frac{1}{\hat{\beta}_{j i}}}(t)\right| \leq \frac{N_{n+j}(\varepsilon)}{\hat{\beta}_{j j}} \rho^{\frac{1}{\hat{\beta}_{j j}}}\left|y_{n+j}(t)-\bar{y}_{n+j}(t)\right|,
$$

where $i=1,2, \ldots, n, j=1,2, \ldots, m$,

$$
\begin{aligned}
& N_{i}(\varepsilon)=\max \left\{\left(\frac{1}{\rho}\left(l_{i}^{-}\right)^{\alpha_{i i}}-\varepsilon\right)^{\frac{1-\alpha_{i i}}{\alpha_{i i}}},\left(\frac{1}{\rho}\left(l_{i}^{+}\right)^{\alpha_{i i}}+\varepsilon\right)^{\frac{1-\alpha_{i i}}{\alpha_{i i}}}\right\}, \\
& N_{n+j}(\varepsilon)=\max \left\{\left(\frac{1}{\rho}\left(l_{n+j}^{-}\right)^{\hat{\beta}_{j i}}-\varepsilon\right)^{\frac{1-\hat{\beta}_{j i}}{\hat{\beta}_{j j}}},\left(\frac{1}{\rho}\left(l_{n+j}^{+}\right)^{\hat{\beta}_{j i}}+\varepsilon\right)^{\frac{1-\hat{\beta}_{j i j}}{\hat{\beta}_{j j}}}\right\} .
\end{aligned}
$$

Letting $\varepsilon \rightarrow 0^{+}$in (4.28)-(4.29), and employing (4.27)-(4.29), we obtain

$$
\left|x_{h}(t)-\bar{x}_{h}(t)\right|<M^{*} e^{-\mu t}, \quad h=1,2, \ldots, n+m, t>0,
$$


where $M^{*}=M \max _{1 \leq i \leq n, 1 \leq j \leq m}\left\{\frac{N_{i}(0)}{\alpha_{i i}} \rho^{\frac{1}{\alpha_{i i}}}, \frac{N_{n+j}(0)}{\hat{\beta}_{j j}} \rho^{\frac{1}{\hat{\beta_{j j}}}}\right\}$. Thus, we have proved that the unique positive almost periodic solution $\left(\bar{x}_{1}(t), \bar{x}_{2}(t), \ldots, \bar{x}_{n+m}(t)\right)^{T}$ of system (1.4) is globally exponentially stable. The proof of Theorem 4.1 is complete.

\section{Illustrative example}

Consider the following two-layer Gilpin-Ayala predator-prey model with time delays:

$$
\left\{\begin{aligned}
x_{1}^{\prime}(t)= & x_{1}(t)\left[r(t)-a(t) x_{1}^{\alpha}(t)-b(t) x_{2}^{\beta}(t)-c(t) x_{1}^{\gamma}(t-\tau(t))\right. \\
& \left.-d(t) x_{2}^{\delta}(t-\sigma(t))\right], \\
x_{2}^{\prime}(t)= & x_{2}(t)\left[-\hat{r}(t)+\hat{a}(t) x_{1}^{\hat{\alpha}}(t)-\hat{b}(t) x_{2}^{\hat{\beta}}(t)+\hat{c}(t) x_{1}^{\hat{\gamma}}(t-\hat{\tau}(t))\right. \\
& \left.-\hat{d}(t) x_{2}^{\hat{\delta}}(t-\hat{\sigma}(t))\right],
\end{aligned}\right.
$$

where $\alpha=\frac{3}{4}, \beta=\frac{1}{2}, \gamma=\frac{1}{3}, \delta=\frac{1}{4}, \hat{\alpha}=3, \hat{\beta}=4, \hat{\gamma}=\frac{3}{2}, \hat{\delta}=1, r(t)=10+\cos \sqrt{2} t, \hat{r}(t)=$ $\frac{2+\sin \sqrt{3} t}{20}, a(t)=6+\sin \sqrt{2} t, b(t)=\frac{2-\sin \sqrt{3} t}{10}, c(t)=\frac{1+|\sin \sqrt{2} t|}{10}, d(t)=\frac{2+\cos \sqrt{5} t}{10}, \hat{a}(t)=\frac{3-\cos \sqrt{2} t}{5}$, $\hat{b}(t)=\frac{7+\sin \sqrt{7} t}{2}, \hat{c}(t)=\frac{1+2|\sin \sqrt{2} t|}{40}, \hat{d}(t)=\frac{2+\cos \sqrt{3} t}{10}$ and

$$
\begin{aligned}
& \tau(t)= \begin{cases}\frac{1+\sin \sqrt{2} t}{4}, & \frac{2 k \pi}{\sqrt{2}}-\frac{\pi}{2 \sqrt{2}} \leq t \leq \frac{2 k \pi}{\sqrt{2}}+\frac{\pi}{2 \sqrt{2}}, k \in \mathbb{Z}, \\
\frac{1-\sin \sqrt{2} t}{4}, & \frac{2 k \pi}{\sqrt{2}}+\frac{\pi}{2 \sqrt{2}} \leq t \leq \frac{2 k \pi}{\sqrt{2}}+\frac{3 \pi}{2 \sqrt{2}}, k \in \mathbb{Z},\end{cases} \\
& \sigma(t)= \begin{cases}\frac{1+\sin \sqrt{3} t}{3}, & \frac{2 k \pi}{\sqrt{3}}-\frac{\pi}{2 \sqrt{3}} \leq t \leq \frac{2 k \pi}{\sqrt{3}}+\frac{\pi}{2 \sqrt{3}}, k \in \mathbb{Z}, \\
\frac{1-\sin \sqrt{3} t}{3}, & 2 \frac{k \pi}{\sqrt{3}}+\frac{\pi}{2 \sqrt{3}} \leq t \leq \frac{2 k \pi}{\sqrt{3}}+\frac{3 \pi}{2 \sqrt{3}}, k \in \mathbb{Z},\end{cases} \\
& \hat{\tau}(t)= \begin{cases}\frac{1-\cos \sqrt{2} t}{3}, & \frac{2 k \pi}{\sqrt{2}} \leq t \leq \frac{2 k \pi}{\sqrt{2}}+\frac{\pi}{\sqrt{2}}, k \in \mathbb{Z}, \\
\frac{1+\cos \sqrt{2} t}{3}, & \frac{2 k \pi}{\sqrt{2}}+\frac{\pi}{\sqrt{2}} \leq t \leq \frac{2 k \pi}{\sqrt{2}}+\frac{2 \pi}{\sqrt{2}}, k \in \mathbb{Z},\end{cases} \\
& \hat{\sigma}(t)= \begin{cases}\frac{2-\cos \sqrt{3} t}{4}, & \frac{2 k \pi}{\sqrt{3}} \leq t \leq \frac{2 k \pi}{\sqrt{3}}+\frac{\pi}{\sqrt{3}}, k \in \mathbb{Z}, \\
\frac{2+\cos \sqrt{3} t}{4}, & 2 \frac{k \pi}{\sqrt{3}}+\frac{\pi}{\sqrt{3}} \leq t \leq \frac{2 k \pi}{\sqrt{3}}+\frac{2 \pi}{\sqrt{3}}, k \in \mathbb{Z} .\end{cases}
\end{aligned}
$$

Obviously, $r(t), \hat{r}(t), a(t), b(t), c(t), d(t), \hat{a}(t), \hat{b}(t), \hat{c}(t), \hat{d}(t), \tau(t), \sigma(t), \hat{\tau}(t)$ and $\hat{\sigma}(t)$ are all positive almost periodic functions. By simple calculation, we have $r^{M}=11, r^{l}=9, \hat{r}^{M}=\frac{3}{20}$, $\hat{r}^{l}=\frac{1}{20}, a^{M}=7, a^{l}=5, b^{M}=\frac{3}{10}, b^{l}=\frac{1}{10}, c^{M}=\frac{1}{5}, c^{l}=\frac{1}{10}, d^{M}=\frac{3}{10}, d^{l}=\frac{1}{10}, \hat{a}^{M}=\frac{4}{5}, \hat{a}^{l}=\frac{2}{5}$, $\hat{b}^{M}=4, \hat{b}^{l}=3, \hat{c}^{M}=\frac{3}{40}, \hat{c}^{l}=\frac{1}{40}, \hat{d}^{M}=\frac{3}{10}, \hat{d}^{l}=\frac{1}{10}$ and

$$
\begin{aligned}
& l_{1}^{+}=\left(\frac{r^{M}}{a^{l}}\right)^{\frac{1}{\alpha}}=\left(\frac{11}{2}\right)^{\frac{4}{3}} \approx 2.8613, \\
& l_{2}^{+}=\left(\frac{\hat{a}^{M}\left(l_{1}^{+}\right)^{\hat{\alpha}}+\hat{c}^{M}\left(l_{1}^{+}\right)^{\hat{\gamma}}}{\hat{b}^{l}}\right)^{\frac{1}{\hat{\beta}}} \approx 1.590, \\
& l_{1}^{-}=\left(\frac{r^{l}-b^{M}\left(l_{2}^{+}\right)^{\beta}-c^{M}\left(l_{1}^{+}\right)^{\gamma}-d^{M}\left(l_{2}^{+}\right)^{\delta}}{a^{M}}\right)^{\frac{1}{\alpha}} \approx 1.1951, \\
& l_{2}^{-}=\left(\frac{\hat{a}^{l}\left(l_{1}^{-}\right)^{\hat{\alpha}}+\hat{c}^{l}\left(l_{1}^{-}\right)^{\hat{\gamma}}-\hat{r}^{M}-\hat{d}^{M}\left(l_{2}^{+}\right)^{\hat{\delta}}}{\hat{b}^{M}}\right)^{\frac{1}{\hat{\beta}}} \approx 1.1303, \\
& 9=r^{l}>b^{M}\left(l_{2}^{+}\right)^{\beta}+c^{M}\left(l_{1}^{+}\right)^{\gamma}+d^{M}\left(l_{2}^{+}\right)^{\delta} \approx 0.9991, \\
& \hat{a}^{l}\left(l_{1}^{-}\right)^{\hat{\alpha}}+\hat{c}^{l}\left(l_{1}^{-}\right)^{\hat{\gamma}} \approx 0.7155>\hat{r}^{M}+\hat{d}^{M}\left(l_{2}^{+}\right)^{\hat{\delta}} \approx 0.6270 .
\end{aligned}
$$


Thus, the assumptions $\left(H_{1}\right)-\left(H_{3}\right)$ are satisfied. So we derive from Theorem 3.1 that system (5.1) has at least one positive almost periodic solution $\left(\bar{x}_{1}(t), \bar{x}_{2}(t)\right)$ satisfying $1.1951 \leq$ $\bar{x}_{1}(t)<2.8613$ and $1.1303 \leq \bar{x}_{2}(t)<1.590$.

In addition, obviously, $0<1-\tau^{\prime}(t)=1-\frac{\sqrt{2}|\cos \sqrt{2} t|}{4}<1,0<1-\sigma^{\prime}(t)=1-\frac{\sqrt{3}|\cos \sqrt{3} t|}{3}<1$, $0<1-\hat{\tau}^{\prime}(t)=1-\frac{\sqrt{2}|\sin \sqrt{2} t|}{3}<1,0<1-\hat{\sigma}^{\prime}(t)=1-\frac{\sqrt{3}|\sin \sqrt{3} t|}{4}<1, \alpha=\frac{3}{4}>\max \{\beta, \gamma, \delta\}=$ $\max \left\{\frac{1}{2}, \frac{1}{3}, \frac{1}{4}\right\}=\frac{1}{2}, \hat{\beta}=4>\max \{\hat{\alpha}, \hat{\gamma}, \hat{\delta}\}=\max \left\{3, \frac{3}{2}, 1\right\}=3$. Take $\rho=1<\min \left\{l_{1}^{-}, l_{2}^{-}\right\} \approx 1.1303$, we have

$$
\begin{aligned}
& -\alpha \rho a^{l}+\hat{\beta} \rho^{\frac{\hat{\alpha}}{\hat{\beta}}} \hat{a}^{M}+\alpha \rho^{\frac{\gamma}{\alpha}} c^{M}+\hat{\beta} \rho^{\hat{\frac{\gamma}{\hat{\beta}}}} \hat{c}^{M}=-0.10<0, \\
& -\hat{\beta} \rho \hat{b}^{l}+\alpha \rho^{\frac{\beta}{\alpha}} b^{M}+\alpha \rho^{\frac{\delta}{\alpha}} d^{M}+\hat{\beta} \rho^{\frac{\hat{\delta}}{\hat{\beta}}} \hat{d}^{M}=-10.35<0 .
\end{aligned}
$$

Thus we verify that $\left(H_{4}\right)-\left(H_{6}\right)$ hold. Therefore, according to Theorem 4.1, we conclude that the unique positive almost periodic solution $\left(\bar{x}_{1}(t), \bar{x}_{2}(t)\right)$ of system (5.1) is globally exponentially stable.

\section{Conclusions}

Compared with the Lotka-Volterra models, the Gilpin-Ayala models are more advantageous because the rate of change in the size of each species is a nonlinear function of the sizes of the interacting species in the Gilpin-Ayala models and the rate of change in the size of each species is a linear function of the sizes of the interacting species the LotkaVolterra models. It is more precise to describe some ecosystems by the Gilpin-Ayala models than by the Lotka-Volterra models. Therefore, we mainly study a class of two-layer Gilpin-Ayala predator-prey model with time delays in this paper. By means of Mawhin's continuation theorem of coincidence degree theory, we obtain some new sufficient conditions of the existence of positive almost periodic solutions. We also obtain the global exponential stability of the positive almost periodic solution for this system by constructing appropriate Lyapunov functionals and smart transformations. Our results provide a theoretical basis for the detection and artificial control of some ecological systems with periodic or almost periodic phenomena.

\section{Acknowledgements}

The author would like to thank the anonymous referees for their useful and valuable suggestions. This work was supported by the National Natural Sciences Foundation of Peoples Republic of China under Grant (No. 11161025; No. 11661047).

Competing interests

The author declares to have no competing interests.

Authors' contributions

The author read and approved the final manuscript.

\section{Publisher's Note}

Springer Nature remains neutral with regard to jurisdictional claims in published maps and institutional affiliations.

Received: 24 January 2018 Accepted: 1 April 2018 Published online: 10 April 2018

\section{References}

1. Ayala, F., Gilpin, M., Eherenfeld, J.: Competition between species: theoretical models and experimental tests. Theor. Popul. Biol. 4, 331-356 (1973)

2. Gilpin, M., Ayala, F.: Global models of growth and competition. Proc. Natl. Acad. Sci. USA 70, 3590-3593 (1973)

3. Chen, L.: Mathematical Models and Methods in Ecology. Science Press, Beijing (1988) (in Chinese) 
4. Chen, F., Chen, Y., Guo, S., Zhong, L.: Global attractivity of a generalized Lotka-Volterra competition model. Differ. Equ. Dyn. Syst. 18(3), 303-315 (2010)

5. Li, C., Lu, S.: The qualitative analysis of $\mathrm{N}$-species periodic coefficient, nonlinear relation, prey-competition systems. Appl. Math. J. Chin. Univ. Ser. A 12, 147-156 (1997)

6. Chen, F.: Some new results on the permanence and extinction of nonautonomous Gilpin-Ayala type competition model with delays. Nonlinear Anal., Real World Appl. 7(5), 1205-1222 (2006)

7. He, M. Li, Z., Chen, F.: Permanence, extinction and global attractivity of the periodic Gilpin-Ayala competition system with impulses. Nonlinear Anal., Real World Appl. 11, 1537-1551 (2010)

8. Wang, D.: Dynamic behaviors of an obligate Gilpin-Ayala system. Adv. Differ. Equ. 2016, 270 (2016)

9. Lu, H., Yu, G.: Permanence of a Gilpin-Ayala predator-prey system with time-dependent delay. Adv. Differ. Equ. 2015, 109 (2015).

10. Lian, B., Hu, S.: Asymptotic behaviour of the stochastic Gilpin-Ayala competition models. J. Math. Anal. Appl. 339, 419-428 (2008)

11. Ai, X., Sun, Y:: An optimal stopping problem in the stochastic Gilpin-Ayala population model. Adv. Differ. Equ. 2012, 210 (2012)

12. Fang, H.: Multiple positive periodic solutions for a food-limited two-species Gilpin-Ayala competition patch system with periodic harvesting terms. J. Inequal. Appl. 2012, 291 (2012)

13. Fang, H., Wang, Y.: Four periodic solutions for a food-limited two-species Gilpin-Ayala type predator-prey system with harvesting terms on time scales. Adv. Differ. Equ. 2013, 278 (2013)

14. Zhang, S., Meng, X., Feng, T., Zhang, T.: Dynamics analysis and numerical simulations of a stochastic non-autonomous predator-prey system with impulsive effects. Nonlinear Anal. Hybrid Syst. 26, 19-37 (2017)

15. Liu, G., Wang, X., Meng, X., Gao, S.: Extinction and persistence in mean of a novel delay impulsive stochastic infected predator-prey system with jumps. Complexity 2017, Article ID 1950970 (2017)

16. Liu, L., Meng, X.: Optimal harvesting control and dynamics of two species stochastic model with delays. Adv. Differ. Equ. 2017, 18 (2017)

17. He, C.: Almost Periodic Differential Equations. Higher Education Publishing House, Beijing (1992) (in Chinese)

18. Li, Y., Ye, Y.: Multiple positive almost periodic solutions to an impulsive non-autonomous Lotka-Volterra predator-prey system with harvesting terms. Commun. Nonlinear Sci. Numer. Simul. 18, 3190-3201 (2013)

19. Gaines, R., Mawhin, J.: Coincidence Degree and Nonlinear Differential Equations. Springer, Berlin (1977)

20. Gopalsamy, K.: Stability and Oscillations in Delay Differential Equations of Population Dynamics. Kluwer Academic, Dordrecht (1992)

\section{Submit your manuscript to a SpringerOpen ${ }^{\circ}$ journal and benefit from:}

- Convenient online submission

- Rigorous peer review

- Open access: articles freely available online

- High visibility within the field

- Retaining the copyright to your article

Submit your next manuscript at $\gg$ springeropen.com 